\title{
Olfactory-driven beta band entrainment of limbic circuitry during neonatal development
}

\author{
Johanna K. Kostka \& Ileana L. Hanganu-Opatz \\ Institute of Developmental Neurophysiology, Center for Molecular Neurobiology, \\ University Medical Center Hamburg-Eppendorf, 20251 Hamburg, Germany
}
Corresponding authors: Ileana L. Hanganu-Opatz hangop@zmnh.uni-hamburg.de
Johanna K. Kostka
johanna.kostka@zmnh.uni-hamburg.de

Figures: 6

Supplementary Material: 5 Figures, 12 Tables

Number of pages: 52

Number of words in Abstract: 159

Number of words in Introduction: 579

Number of words in Discussion: 1089 
31

32

33

34

35

\section{ABSTRACT}

Cognitive processing relies on the functional refinement of the limbic circuitry during the first two weeks of life. During this developmental period, when most sensory systems are still immature, the sense of olfaction acts as "door to the world", providing the main source of environmental inputs. However, it is unknown whether early olfactory processing shapes the activity in the limbic circuitry during neonatal development. Here, we address this question by combining simultaneous in vivo recordings from the olfactory bulb $(\mathrm{OB})$, lateral entorhinal cortex (LEC), hippocampus (HP), and prefrontal cortex (PFC) with opto- and chemogenetic manipulations of mitral/tufted cells (M/TCs) in the OB of non-anesthetized neonatal mice. We show that the neonatal $\mathrm{OB}$ synchronizes the limbic circuity in beta frequency range. Moreover, it drives neuronal and network activity in LEC, as well as subsequently, HP and PFC via longrange projections from mitral cells (MCs) to HP-projecting LEC neurons. Thus, OB activity shapes the communications within limbic circuits during neonatal development.

(1)

(1)

(1)

8

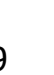

50

\section{1}




\section{INTRODUCTION}

Coordinated neuronal activity during early development refines the neural circuits that account for complex processing in the adult brain. During the first two postnatal weeks, when rodents are still blind, deaf, and perform no active whisking, coordinated activity patterns in the sensory periphery occur independently of sensory input [1,2]. Spontaneous neuronal discharges in the retina, cochlea, and whisker pad trigger discontinuous oscillatory bursts in the corresponding primary sensory cortices [3-7] that are necessary for the development of sensory discrimination [8]. Similar activity patterns can also be observed in brain areas involved in higher cognitive processing. Discontinuous theta band oscillations in the LEC entrain similar activity patterns in the HP, which in turn entrains the prelimbic area $(P L)$ of the PFC [9-12]. Disturbance of these early activity patterns in mouse models of psychiatric risk [13-17] as well as through pharmacological [18] or optogenetic manipulations [19] led to disruption of adult circuits and behavioral abilities. However, it is not clear whether stimulus-independent activity patterns in the sensory periphery impact the limbic network during neonatal development.

Due to the limited or absent functionality of most sensory systems during the first two postnatal weeks, their contribution to the development of limbic networks has been considered negligible. This hypothesis has been supported by data showing that the synchrony between V1 and the HP-PFC network before eye-opening is rather weak [11]. In contrast to other sensory systems, the olfactory system is functional early in life and newborn mice heavily depend on it for survival [20]. Correspondingly, the anatomical pathways from OB to cortical areas are unique among sensory systems. MCs send afferents to the piriform cortex $(\mathrm{PiR})$ and limbic brain areas such as LEC and amygdala, lacking the relay through the thalamus $[21,22]$. At adult age, in line with the anatomical connectivity, strong functional coupling during odor processing has been found between $\mathrm{OB}$ and these brain areas. For example, adult olfactory processing relies on respiration-modulated beta and gamma OB activity [23-25]. Further, beta oscillations in PiR, LEC, and HP play a critical role in olfactory memory processing [26-28]. Moreover, synchronized beta oscillations between OB-HP and LEC-HP are critically involved 
in odor learning [29-32]. Recently, beta oscillations in prefrontal-hippocampal networks have been identified to support the utilization of odor cues for memory-guided decision making [33].

The tight and behaviorally relevant coupling between OB and limbic circuits at adult age leads to the question, which role does olfactory activation early in life play for these circuits. Previously, we showed that discontinuous oscillatory activity in the theta-beta range, emerging as a result of bursting MCs in the neonatal $\mathrm{OB}$, entrains similar oscillatory patterns in LEC $[34,35]$. However, the role of neuronal and network activity in the OB for the functional entrainment of downstream areas within neonatal limbic circuits is still largely unknown.

To address this knowledge gap, we simultaneously monitored single-unit activity (SUA) and local field potentials (LFP) in OB, LEC, HP, and PFC of non-anesthetized neonatal mice (postnatal day (P) 8-10) during manipulation of $\mathrm{M} / \mathrm{TC}$ activity using excitatory opsins and inhibitory DREADDs. We show that activation of M/TCs triggers action potential firing in LEC and HP as well as prominent beta oscillations that synchronize the OB with the downstream cortical areas. Conversely, blocking MC output specifically diminishes OB - limbic network coupling in beta frequency range. These data document the ability of coordinated activity at the sensory periphery of newborns to shape the network activity in circuits accounting for adult cognitive processing.

\section{RESULTS}

\section{Oscillatory activity in OB times the network activity in limbic circuits of neonatal mice}

To get first insights into the impact of $\mathrm{OB}$ activity on developing cortical circuits including LEC, $\mathrm{HP}$, and PFC, we simultaneously recorded the LFP and multiunit activity (MUA) in all four brain areas in non-anesthetized neonatal (P8-10) mice ( $n=56$, Figs $1 A$ and $B$ ) and assessed the temporal relationships between network oscillations and neuronal firing. All investigated areas showed discontinuous oscillatory activity in theta-beta range $[11,12,34]$, accompanied by continuous low amplitude slow frequency oscillations peaking at 2-4 Hz (respiration rhythm, RR) (Fig 1B). Half (med: $53.779 \%$, iqr: $47.681-65.104 \%, n=20$ ) of the oscillatory events 
detected in $\mathrm{OB}$ co-occurred in all four brain regions. To quantify the coupling of $\mathrm{OB}$ to cortical areas, we calculated the imaginary coherence (Fig 1C). While a high level of synchrony linked OB with all investigated cortical areas, the strength of coupling was frequency-dependent, having the highest magnitude in the beta frequency range for OB-LEC and OB-HP and in the RR frequency band for OB-LEC and OB-PFC.

To uncover whether $\mathrm{OB}$ activity times the neuronal firing of cortical areas, we calculated the phase-locking of single units (SUA) recorded in OB, LEC, HP, and PFC to beta band oscillations (12-30 Hz) in OB (Fig 1D). Significantly locked OB units fired shortly before the trough of the beta cycle, while LEC and HP units were locked to significantly shifted phase angles (Fig 1D, S1 and S2 Tables). Solely the prefrontal firing showed no phase preference of locking to the oscillatory phase in $\mathrm{OB}$. Next, we questioned whether the communication between $\mathrm{OB}$ and cortical areas is directed and whether $\mathrm{OB}$ acts as a driving force within the circuit. For this, we assessed the temporal relationship between the firing in cortical regions and $\mathrm{OB}$ by calculating the standardized cross-covariance of unit pairs [36]. For unit pairs between $\mathrm{OB}$ and LEC, OB and HP, and $\mathrm{OB}$ and PFC, the peak of cross-covariance was at negative time-lags, indicating that spiking in OB preceded cortical firing (Fig 1E). Monitoring the timing of interactions between cortical areas (LEC-HP, LEC-PFC, and HP-PFC) confirmed the previously reported directionality of communication [12], yet less clear as for the OB-driven coupling. As spike-dependent methods are strongly biased by the firing rate of investigated neurons, which is rather low in neonatal mice, we next used the spectral dependency ratio (SDR), a method that infers causal direction from time-series data $[37,38]$, to confirm the directed communication between OB and cortical areas. SDR values for OB $\rightarrow$ LEC were significantly higher than for LEC $\rightarrow O B$, supporting the drive from OB to LEC. Further, the SDR analysis revealed a spectral dependency of $\mathrm{HP}$ as well as PFC on $\mathrm{OB}$, suggesting the contribution of $\mathrm{OB}$ activity to the oscillatory entrainment of prefrontal and hippocampal circuits (Fig 1F and S3 Table). Moreover, the analysis confirmed the previously reported directed interaction from HP to PFC and LEC to PFC $[11,12]$. No SDR difference was detected for 
137 LEC-HP, indicating that, in line with anatomical data [12], a bidirectional coupling links HP and 138 LEC (Fig 1F and S3 Table).

Thus, tight directed interactions between $\mathrm{OB}$ and cortical areas ensure timed firing and oscillatory entrainment within downstream LEC-HP circuits. 
A
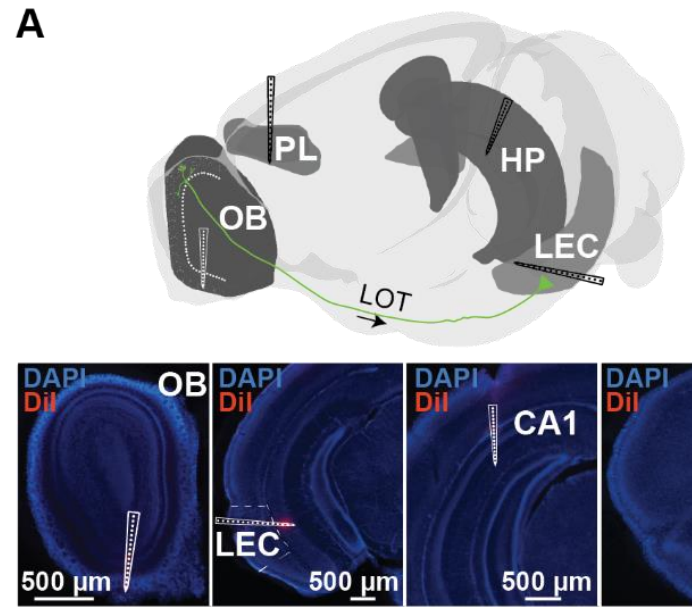

C

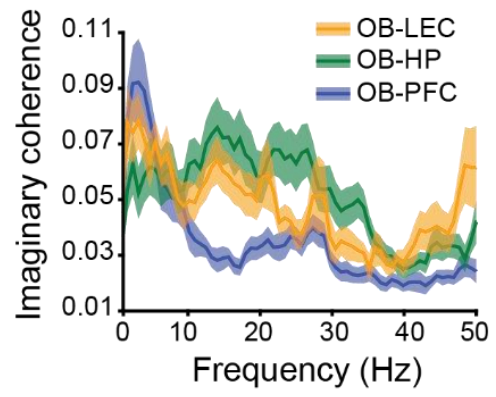

$500 \mu \mathrm{m}$

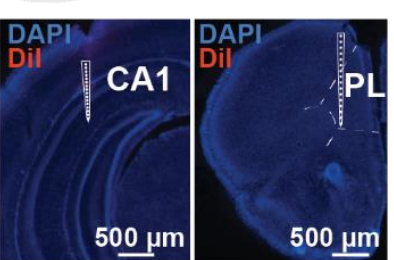

D

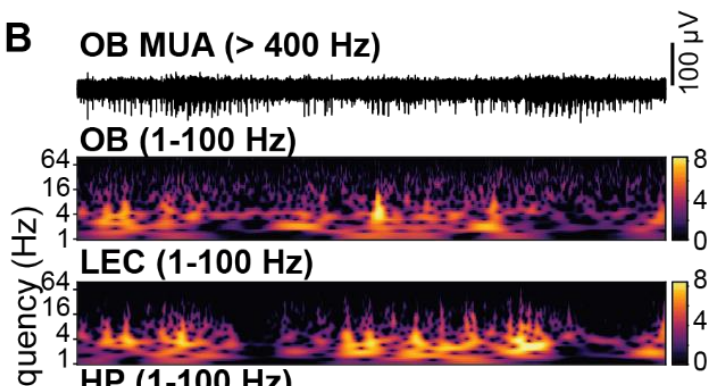

要 $\mathrm{HP}(1-100 \mathrm{~Hz})$

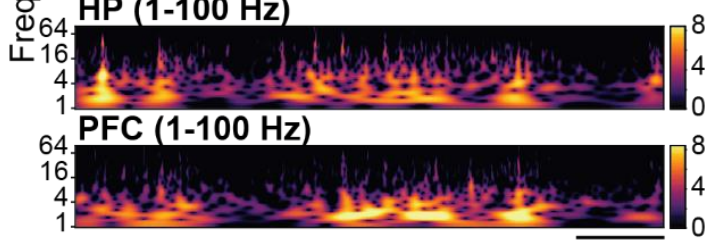

Time (s)

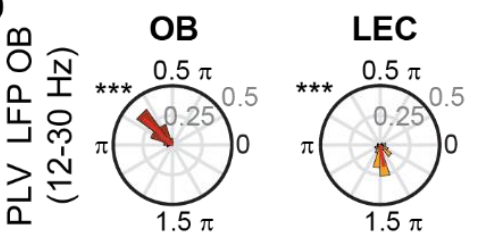

HP

PFC
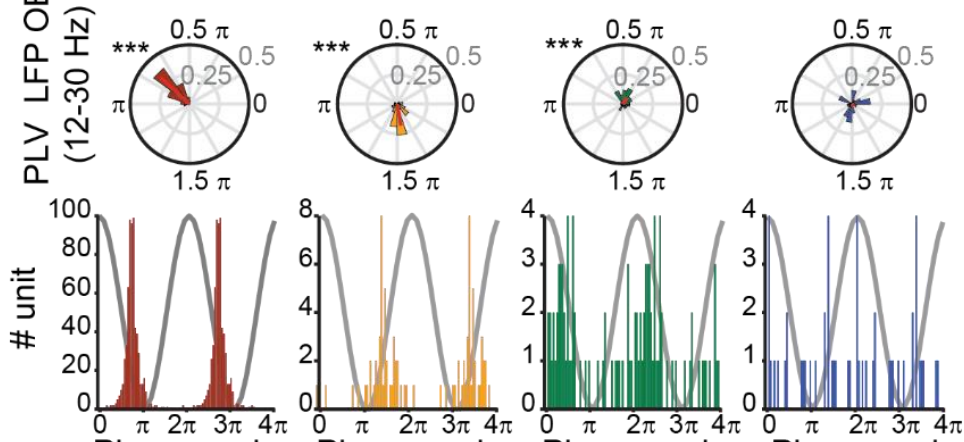

Phase angle

$\mathbf{F}$
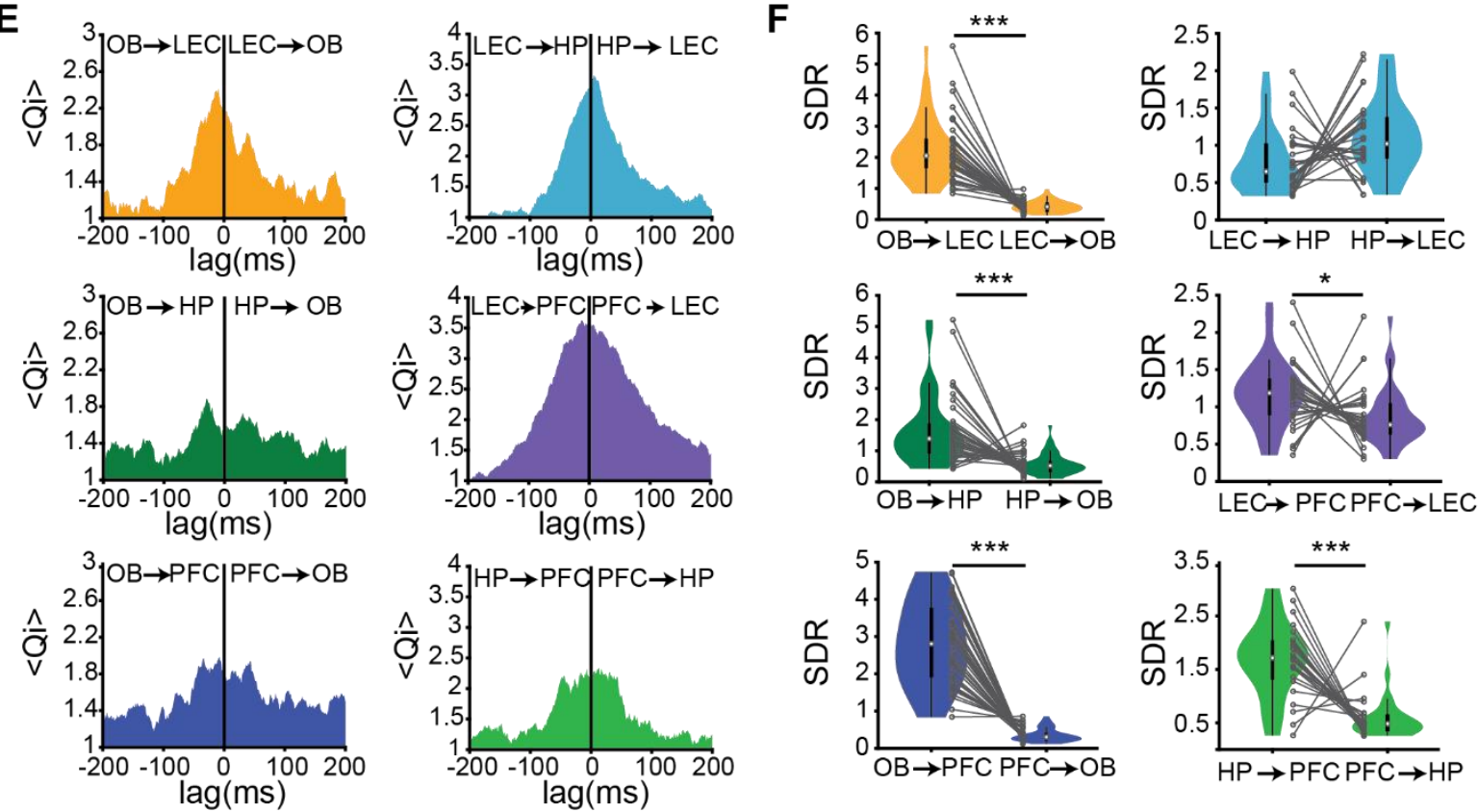

Fig 1. Functional coupling between neonatal OB, LEC, HP, and PFC

(A) Top, schematic of recording configuration for simultaneous extracellular recordings in OB, LEC, HP, and PFC. The positions of recording sites were displayed superimposed on the corresponding brain areas (Brainrender: [39]). Bottom, digital photomontages displaying the Dil-labeled (red) electrode tracks in DAPI (blue) stained slices of OB, LEC, HP, and PL of a P10 mouse. (B) Representative MUA trace 
recorded in the mitral cell layer $(\mathrm{MCL})$ displayed together with the wavelet spectra of LFP recorded simultaneously in OB, LEC, HP, and PFC. (C) Spectra of imaginary coherence calculated for OB - LEC (yellow), OB - HP (green), and OB - PFC (blue). (D) Top, polar plots displaying the phase-locking of significantly locked units in OB (red), LEC (yellow), HP (green), and PFC (blue) to beta oscillations in OB. Bottom, histograms of mean phase angle for significantly phase-locked OB (red), LEC (yellow), HP (green), and PFC (blue) units. Histograms are replicated over two OB beta cycles (gray curve). ${ }^{* * *} \mathrm{p}<$ 0.001 , Rayleigh test for non-uniformity). (E) Plots of standardized mean spike-spike cross-covariance for OB - LEC (yellow), OB - HP (green), OB - PFC (blue), LEC - HP (light blue), LEC - PFC (purple), and HP - LEC (light green). Negative lags indicate that spiking in the first brain area precedes spiking in the second brain area. (F) Spectral dependency ratio (SDR) calculated for OB - LEC (yellow), OB - HP (green), OB - PFC (blue), LEC - HP (light blue), LEC - PFC (purple), and HP - LEC (light green). Gray dots and lines correspond to individual animals. $\left({ }^{*} p<0.05\right.$, ${ }^{* *} p<0.01,{ }^{* * *} p<0.001$, Wilcoxon signedrank test).

\section{Activation of M/TCs induces beta oscillations in neonatal OB}

To elucidate the mechanisms of directed communication between $\mathrm{OB}$ and downstream cortical areas, we activated ChR2-transfected M/TCs by light and simultaneously monitored the network and neuronal activity in neonatal LEC, HP, and PFC. Transfection of M/TCs was achieved using a cre-dependent virus vector (AAV9-Ef1a-DIO-hChR2(E123T/T159C)-EYFP) that was injected into the right OB of P1 Tbet-cre mice (Fig 2A). ChR2-EYFP expression was reliably detected in M/TCs and their projections 7 days after injection (Fig 2B). Ramp light stimuli of increasing intensity (473 nm, total duration $3 \mathrm{~s}$ ) were used to activate M/TCs in the OB of P8-10 mice (Fig 2A). The stimulation parameters have been set in line with previous data [40] to prevent not only firing as a result of tissue heating but also artificially synchronous firing patterns and large stimulation artifacts. Ramp stimulation led to a sustained increase of spike discharge and broad-band (4-100 Hz) LFP power augmentation in OB that peaked in beta frequency range $(12-30 \mathrm{~Hz})$ (Figs 2C, 2D, and S1A Fig). In cre $^{+}$mice, the modulation indices $(\mathrm{Ml})$ for theta, beta, and gamma power were significantly increased and different from those calculated for cre- animals (Fig 2Dii and S5 Table). Correspondingly, SUA strongly augmented during ramp stimulation (Figs $2 \mathrm{E}$ and $2 \mathrm{~F}$ ). This activation was not layer-specific and, mirroring the tight $\mathrm{OB}$ wiring, not only M/TCs but also granule cells (GCs) and other OB 
interneurons increased their firing in response to light activation of ChR2-transfected M/TCs (S1B and S1C Figs). Analysis of the firing onset along OB layers confirmed the global activation. Cells in the MCL and GCL started to fire immediately after the 3 ms-long light pulses, whereas cells in the extra plexiform layer (EPL) and glomerular layer $(\mathrm{GL})$ responded with a brief delay (Fig 2G).

To assess the temporal relationship between neuronal firing and beta oscillations in $\mathrm{OB}$, we calculated the locking of SUA firing to the oscillatory phase before (Pre) and during (Stim) light stimulation. Ramp stimulation caused a significantly stronger locking of OB units to beta oscillations (Pre: med: 0.147 , iqr: $0.094-0.227$; Stim: med: 0.180 , iqr: $0.103-0.294$, $\mathrm{n}_{\text {units }}=176$ from 26 mice, $\mathrm{p}=9.39^{*} 10^{-5}$, LMEM) (Fig $\left.2 \mathrm{Hi}\right)$ and an augmentation of the proportion of significantly phase-locked units to the beta rhythm during ramp stimulation (Pre: $16.478 \%$, 29/176 units, Stim: $55.114 \%, 97 / 176$ units, $p=3.12^{*} 10^{-14}$, Fisher's exact test) (Fig 2 Hii). Of note, the coupling of $\mathrm{OB}$ units to the RR phase was weaker (Pre: med: 0.129 , iqr: $0.080-$ 0.220, Stim: med: 0.097 , iqr: $0.057-0.157, n_{\text {units }}=176$ from 26 mice, $p=7.782^{*} 10^{-6}$, LMEM) (S1D Fig) even though the proportion of locked units (Pre: 14.773\%, 26/176 units, Stim: 14.205 $\%, 25 / 176$ units, $p=1$, Fisher's exact test) and the power of RR oscillations were not altered upon light stimulation (S1D(iii) and S2D Figs). In contrast, light stimulation had no effects on the phase-locking of OB units to oscillatory phase in cre- mice (S1E Fig and S4 Table). The larger beta power observed during ramp stimulation might result from increased M/TC and interneuronal firing, since spike-triggered power (STP) analysis revealed that the ability of OB units to trigger beta power is stronger during ramp stimulation compared to baseline periods (Pre: med: $6.694 \mu \mathrm{V}^{2}$, iqr: 2.291 - $16.447 \mu \mathrm{V}^{2}$; Stim: med: $18.285 \mu \mathrm{V}^{2}$, iqr: 4.437 - 58.407 $\mu \mathrm{V}^{2}$; $\mathrm{n}_{\text {units }}=309$ from 19 mice, $\mathrm{p}=3.16^{*} 10^{-13}$, LMEM) (Fig 2l).

These data indicate that the activation of M/TCs recruits the local circuitry in the $\mathrm{OB}$ and thereby organizes the OB network activity in the beta rhythm. 
A
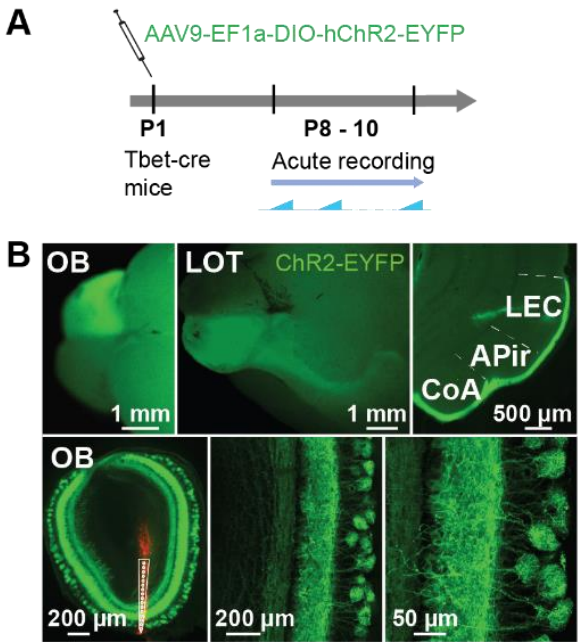

E

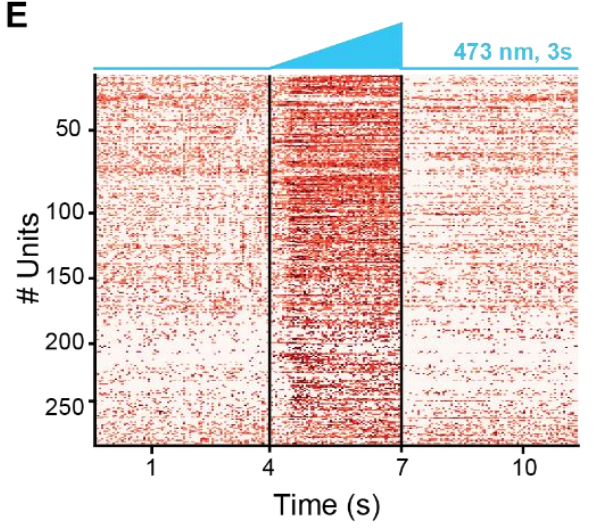

H

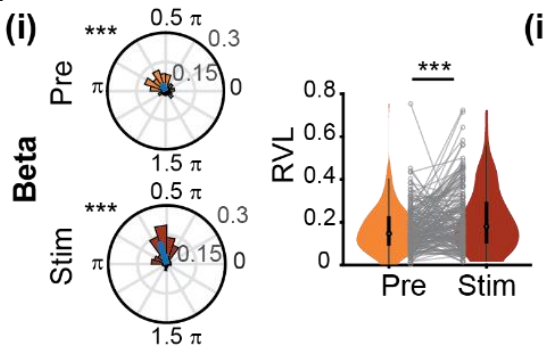

C

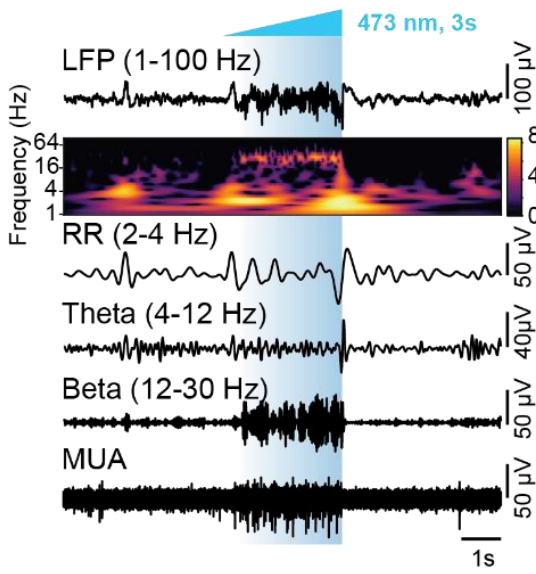

$F_{\text {(i) }}$

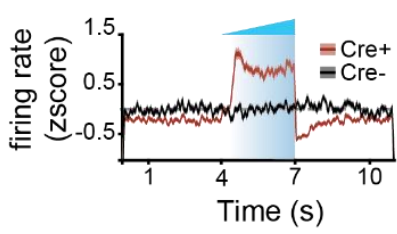

(ii)

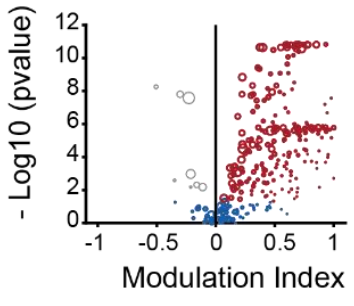

I

(ii)

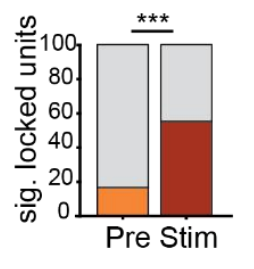

(i)

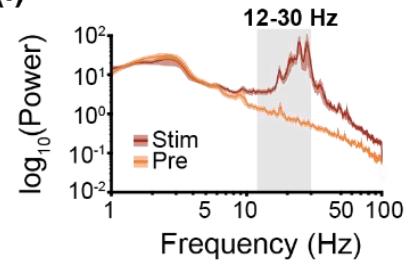

(ii)

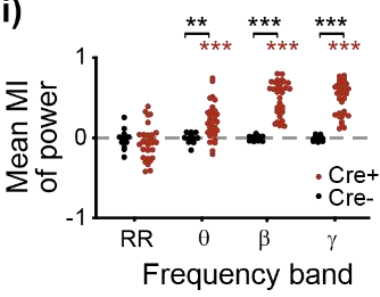

G

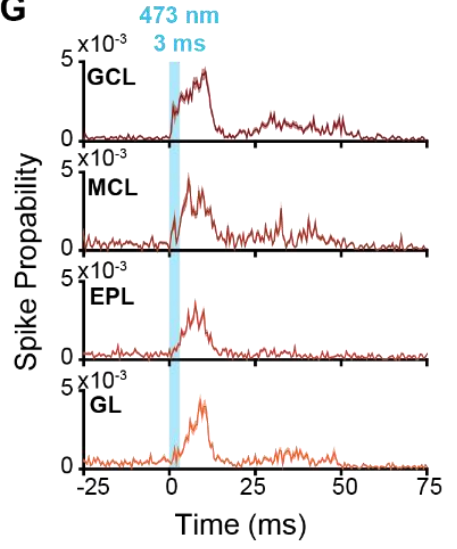

(ii)
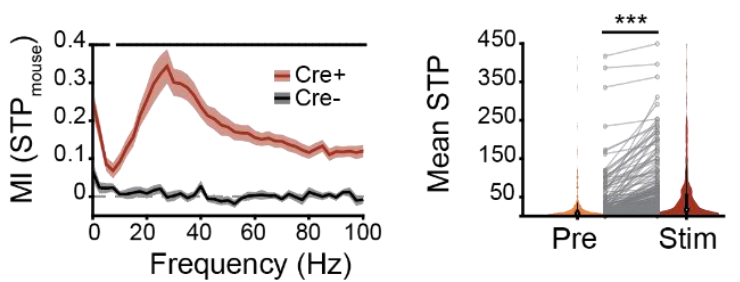

Fig 2. Effects of $M / T C$ manipulation by light on single-unit entrainment and oscillatory activity in OB.

(A) Schematic of the experimental protocol. (B) Top, photograph of the dorsal (left) and ventral side (middle) of a brain from a Tbet-cre ${ }^{+}$mouse showing EYFP expression in the OB and M/TC axonal projections (LOT) to LEC, piriform transition area (APir), and cortical amygdala (CoA) (right). Bottom, digital photomontages displaying the Dil labeled electrode track in $\mathrm{OB}$ (left) and confocal images displaying the mitral cell layer (MCL) of the right $\mathrm{OB}$ at different magnifications (middle and right). (C) Representative extracellularly recorded LFP in the OB displayed band-pass filtered in different frequency bands and accompanied by the corresponding wavelet spectrum during ramp stimulation, as well as by the simultaneously recorded MUA in the MCL. (D) (i) Power spectrum for OB LFP before (orange) and during (red) ramp stimulation. The gray shaded area corresponds to the beta band (12-30 Hz). (ii) Mean MI of LFP power in different frequency bands for $\mathrm{cre}^{+}$(red) and cre- (black) mice. (red stars for cre ${ }^{+}{ }^{* * \star}$ 
$p<0.001$, Wilcoxon signed-rank test; black stars for comparison cre ${ }^{+}$vs. cre: ${ }^{* *} p<0.01,{ }^{* * *} p<0.001$, Wilcoxon rank-sum test). (E) Raster plot of SUA in the OB before, during, and after ramp stimulation. (F) (i) Z-scored firing rate in response to ramp stimulation of units recorded in the $\mathrm{OB}$ of $\mathrm{cre}^{+}$(red) and cre- (black) mice. (ii) MI of SUA firing in response to ramp stimulation (Significantly activated units are shown in red, whereas significantly inhibited units in gray, $p<0.01$, Wilcoxon signed-rank test). (G) Spiking probability of units located in the granule cell layer (GCL), MCL, external plexiform layer (EPL), and glomerular layer (GL) after a $3 \mathrm{~ms}$ light pulse (blue box, $473 \mathrm{~nm}$ ) delivered to the $\mathrm{OB}$. (H) (i) Phase locking of $\mathrm{OB}$ units to beta oscillations in $\mathrm{OB}$. Left, polar plots displaying phase locking of $\mathrm{OB}$ units before (Pre, orange) and during ramp stimulation (Stim, red). The mean resulting vectors are shown as blue lines. ( ${ }^{* * *} p<0.001$, Rayleigh test for non-uniformity). Right, violin plots displaying the resulting vector length (RVL) of $\mathrm{OB}$ units before (Pre, orange) and during ramp stimulation (Stim, red). Gray dots and lines correspond to individual units. ( ${ }^{\star \star *} p<0.001$, linear mixed-effect model). (ii) Percentage of significantly locked units before (Pre, yellow) and during (Stim, red) stimulation. ( ${ }^{* * *} p<0.001$, Fisher's exact test). (I) (i) Plot of mean Ml of spike-triggered power (STP) for $\mathrm{cre}^{+}$(red) and cre- (black) mice during ramp stimulation. (black line: $p<0.05$, Wilcoxon rank-sum test). (ii) Violin plots displaying mean STP for OB units before (Pre, yellow) and during ramp stimulation (Stim, red). Gray dots and lines correspond to individual units. ( ${ }^{* * *} p<0.001$, linear mixed-effect model).

\section{M/TC activation drives neuronal firing in LEC and HP}

To characterize the downstream effects of beta band entrainment of $\mathrm{OB}$, we firstly analyzed the organization of $\mathrm{OB}$ projections in neonatal mice. In line with morphological investigations in adult mice [21], we previously showed that MC axons are present in superficial layers of LEC already at neonatal age [34]. Entorhinal neurons in layer II/III strongly project to HP and weakly to PFC $[12,17]$. Here, we performed axonal tracing of M/TCs using the anterograde virus (AAV9-hSyn-hChR2(H134R)-EYFP) injected into the OB at P8. Simultaneously, we monitored the entorhinal neurons that project to HP by using the retrograde virus (AAVrgCamKIla-mCherry) injected into the HP at P8 (Figs 3A and 3B). At P18, MC axons expressing EYFP were present in layer I/II of LEC and PiR (Fig 3B). Additionally, mCherry-expressing HPprojecting neurons were identified in entorhinal layer II/III. These neurons send their apical dendrites to layer I of LEC, where they collocate with MC axonal projections (Fig 3B). 
circuits. For this, we used pulse $(3 \mathrm{~ms})$ and ramp $(3 \mathrm{~s})$ blue light stimulations $(473 \mathrm{~nm})$ of transfected OB neurons and simultaneously recorded the neuronal activity in LEC, HP, and PFC. Pulse stimulation of M/TCs induced neuronal firing in all investigated brain areas, except PFC (Fig 3Ci). While the light-evoked OB firing rate sharply peaked already 7-8 ms poststimulus, the responses in the other brain areas were substantially broader and delayed (37 $\mathrm{ms}$ in LEC, 45-60 ms in HP). A second firing increase was detected in OB after $\sim 28 \mathrm{~ms}$ and might reflect OB-internal processing or feedback activation from downstream areas. To expand on these results, we used normalized cross-covariance analysis to uncover the temporal correlations between light-evoked spike trains in the investigated brain regions. The most prominent interaction was detected for OB-LEC, with OB firing preceding the entorhinal discharges (Fig 3Cii). While having a similar directionality, the OB-HP cross-covariance peaked later and less precisely. The data gives first insights into the communication pathways relaying the information from M/TCs to LEC and subsequently, to HP.

Ramp stimulation of M/TCs evoked neuronal firing in LEC, HP, and PFC with similar dynamics: a fast increase in OB followed by a delayed spiking in LEC, and subsequently in HP and PFC. In OB, SUA abruptly increased with ramp onset $(76.157 \%$ of units activated significantly, $2.847 \%$ units inhibited significantly) and decreased post-stimulus $(8.185 \%$ of units activated significantly, $29.893 \%$ of units inhibited significantly) (Figs 3Di and 3Ei). In contrast, the average SUA firing rate in LEC, HP, and PFC showed a delayed increase starting around halfway through the ramp and continuing after the light stimulation (Figs 3Dii-iv). Analysis of the proportion of activated units during and after ramp revealed that neurons in downstream areas expressed higher firing rates also after the light was switched off (Fig 3E), indicating that the activation of M/TCs boosted the cortical network activation. Correspondingly, this post-stimulus firing increase recruited more neurons than those activated during ramp stimulation (LEC: $10.959 \%$ during stimulation vs. $21.233 \%$ post-stimulus; HP: $9.167 \%$ vs. $10.833 \%$, PFC: $9.195 \%$ vs. $19.540 \%$ ). In HP, the post-stimulus network effect was not

271 restricted to activation of neurons but also related to the increase in the proportion of neurons 272 that were inhibited after the ramp (7.5\% vs. $11.667 \%)$. Light stimulation of control animals did 
bioRxiv preprint doi: https://doi org/10.1101/2021.10.04.463041; this version posted October 21, 2021. The copyright holder for this preprint (which was not certified by peer review) is the author/funder. All rights reserved. No reuse allowed without permission. and prefrontal circuits.

A

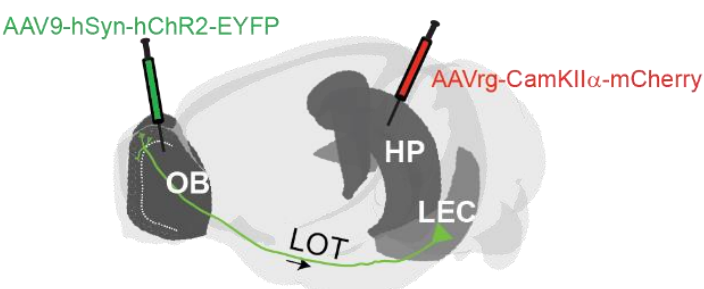

C(i)

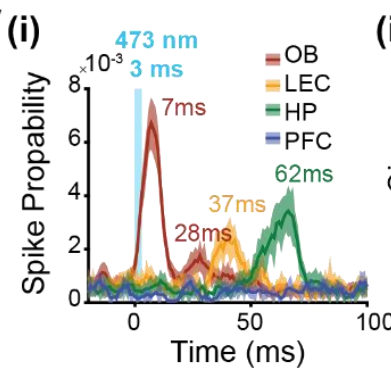

D

(i)

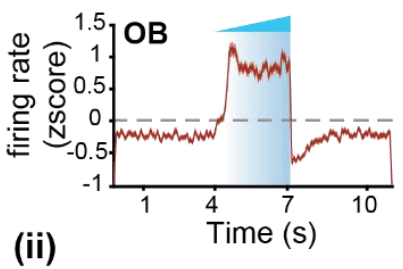

(ii)

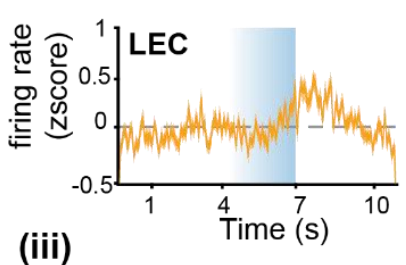

(iii)
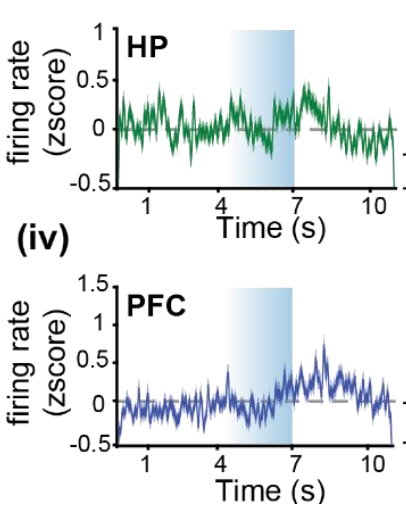

(ii)

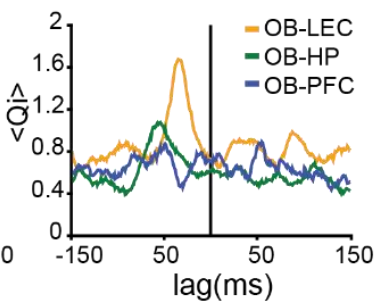

E
Activated units Stim (i)
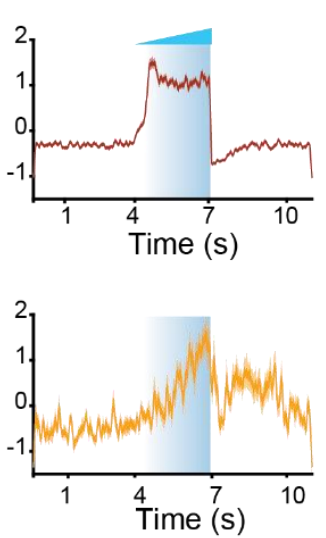
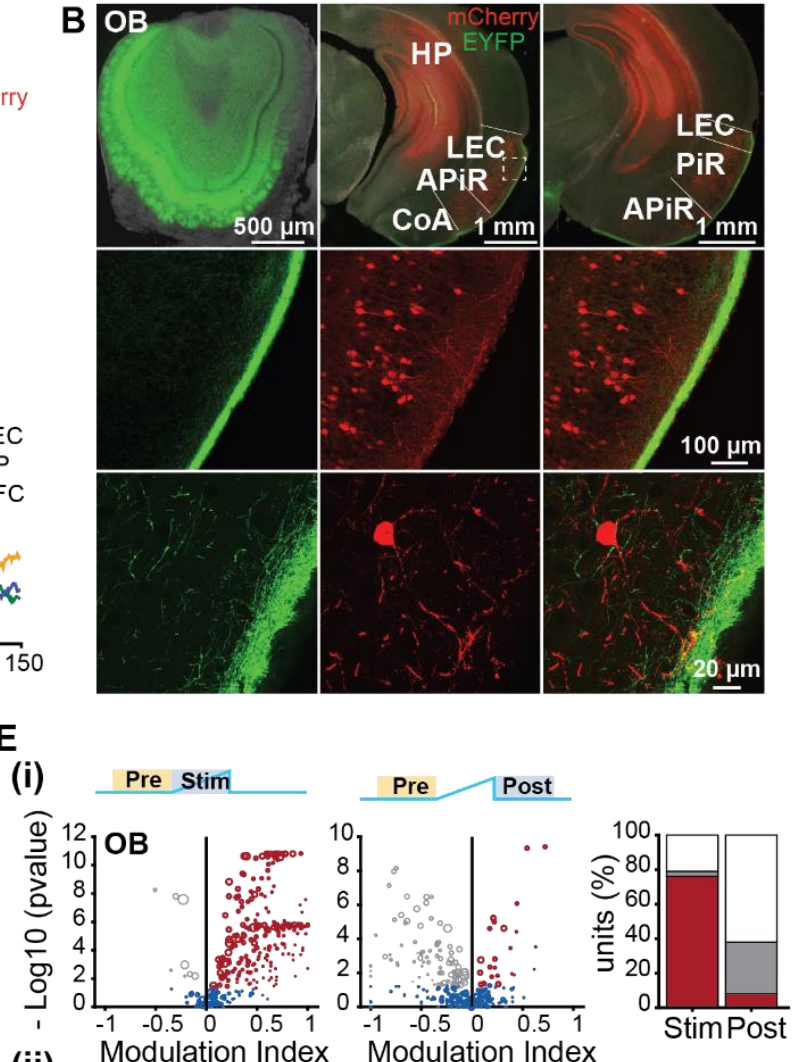

(ii)
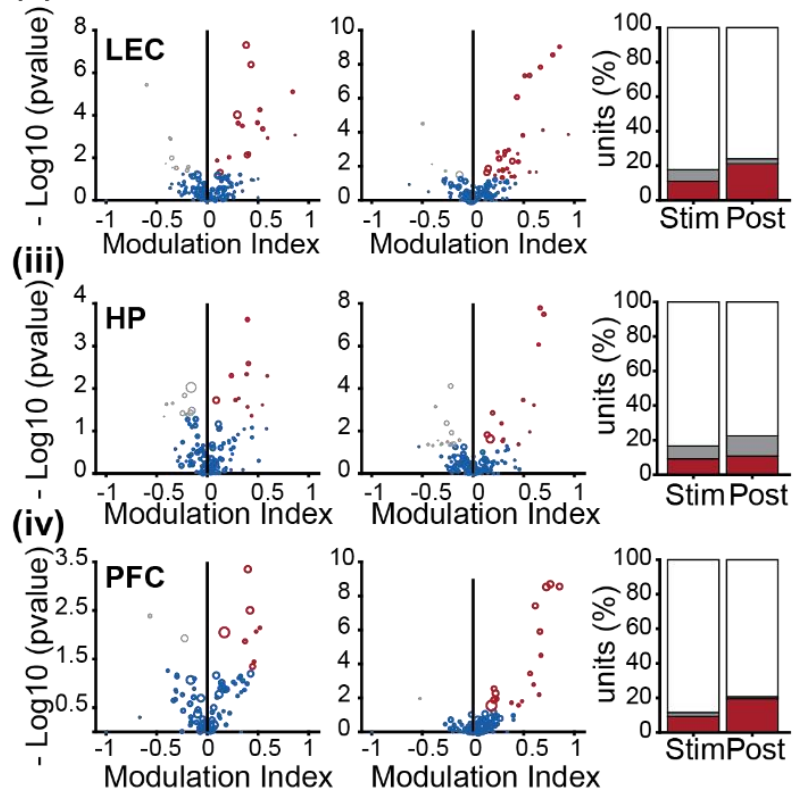

Fig 3. Effects of optogenetic manipulation of M/TCs on single-unit activity in LEC, HP, and PFC. 
bioRxiv preprint doi: https://doi.org/10.1101/2021.10.04.463041; this version posted October 21, 2021. The copyright holder for this preprint (which was not certified by peer review) is the author/funder. All rights reserved. No reuse allowed without permission.

Fig 3. Effects of optogenetic manipulation of M/TCs on single-unit activity in LEC, HP, and PFC.

(A) Schematic of the experimental protocol used to trace MC axons and neurons projecting to HP. (Brainrender: [39]). (B) Top, digital photomontages displaying EYFP (green) and mCherry (red) fluorescence in coronal slices including OB (left, injection side of AAV9-hSyn-hChR2-EYFP), HP (middle, injection site of AAVrg-CamKIl $\alpha$-mCherry), and LEC (right). Note the co-expression of EYFP and mCherry in LEC. Middle, EYFP (left), mCherry (middle), and their co-expression in the LEC are shown at larger magnification (dashed box). Bottom, EYFP (left), mCherry (middle), and their coexpression shown at larger magnification for a HP-projecting entorhinal neuron with dendrites targeting layer I. (C) (i) Spike probability of units in OB (red), LEC (yellow), HP (green), and PFC (blue) after a 3 ms light pulse $(473 \mathrm{~nm})$ delivered to the $\mathrm{OB}$. Numbers indicate the delay of the peak spike probability for each brain area. (ii) Spike-spike cross-covariance for OB - LEC (yellow), OB - HP (green), and OB PFC (blue). Negative lags correspond to OB activity driving spiking in other brain areas. (D) (i) Left, zscored firing rate of units recorded in the $\mathrm{OB}$ of $\mathrm{cre}^{+}$(red) mice in response to light stimulation. Right, $\mathrm{z}$ scored firing rate of significantly activated units during ramp stimulation. (ii) Same as (i) for units recorded in LEC (yellow). (iii) Same as (i) for units recorded in HP (green). (iv) Same as (i) for units recorded in PFC (blue). (E) (i) Left, volcano plot displaying the MI of SUA firing rates recorded in the OB before (Pre) vs. during (Stim) ramp stimulation (significant activated units are shown in red and significant inhibited units in gray, $p<0.01$, Wilcoxon signed-rank test). Middle, same as the left image but for SUA firing rates before (Pre) vs. after (Post) ramp stimulation. Right, bar plots depicting the percentage of activated (red) and inhibited (gray) units during (Stim) and after (Post) ramp stimulation. (ii) Same as (i) for units recorded in LEC. (iii) Same as (i) for units recorded in HP. (iv) Same as (i) for units recorded in PFC.

\section{M/TC activation boosts beta band coupling within downstream limbic circuits}

The long-lasting effects of M/TC stimulation on the neuronal firing of downstream areas, LEC, $\mathrm{HP}$, and PFC suggest that $\mathrm{OB}$ activation might act as a driving force for the generation of network oscillations in neonatal limbic circuits. To test this hypothesis, we paired ramp light stimulation of ChR2-transfected M/TCs with LFP recordings in LEC, HP, and PFC of P8-10 mice. Ramp stimulation of M/TCs increased the oscillatory power in LEC, HP, and PFC (Figs 4A, 4B, and S5 Table). The most prominent increase was detected for beta band oscillations. Moreover, we assessed the degree of synchrony between OB and cortical areas during light stimulation by calculating the imaginary part of coherence, a measure that is insensitive to false connectivity arising from volume conduction [41]. The imaginary coherence between OB 
309 and LEC, OB and HP as well as OB and PFC increased during light activation of M/TCs, the 310 most prominent effects being detected in beta band range (Fig 4C and S6 Table).

312 but also increases the $12-30 \mathrm{~Hz}$ oscillatory coupling between $\mathrm{OB}$ and downstream cortical 313 areas. 


\section{A}

A $\mathrm{OB}$

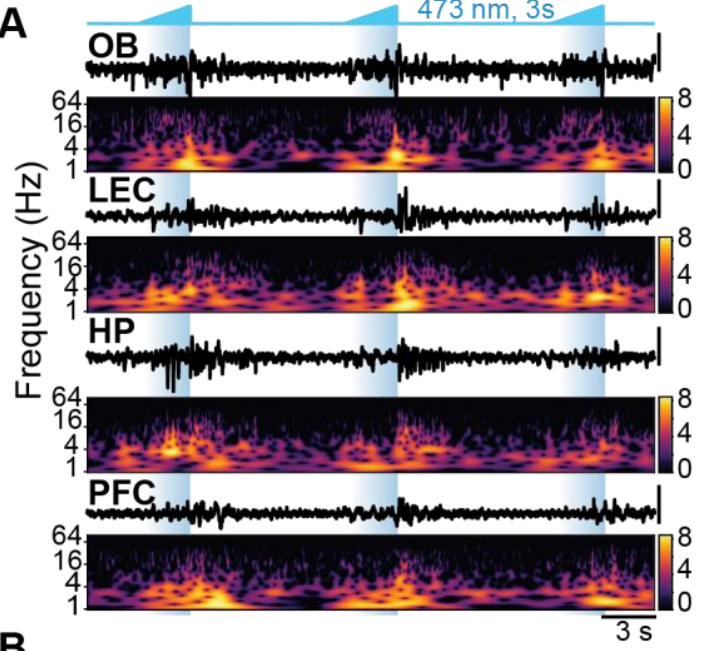

B(i)
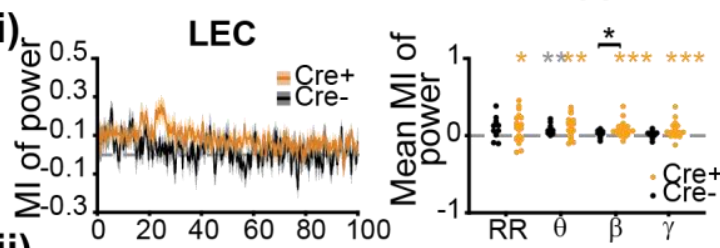

(ii)
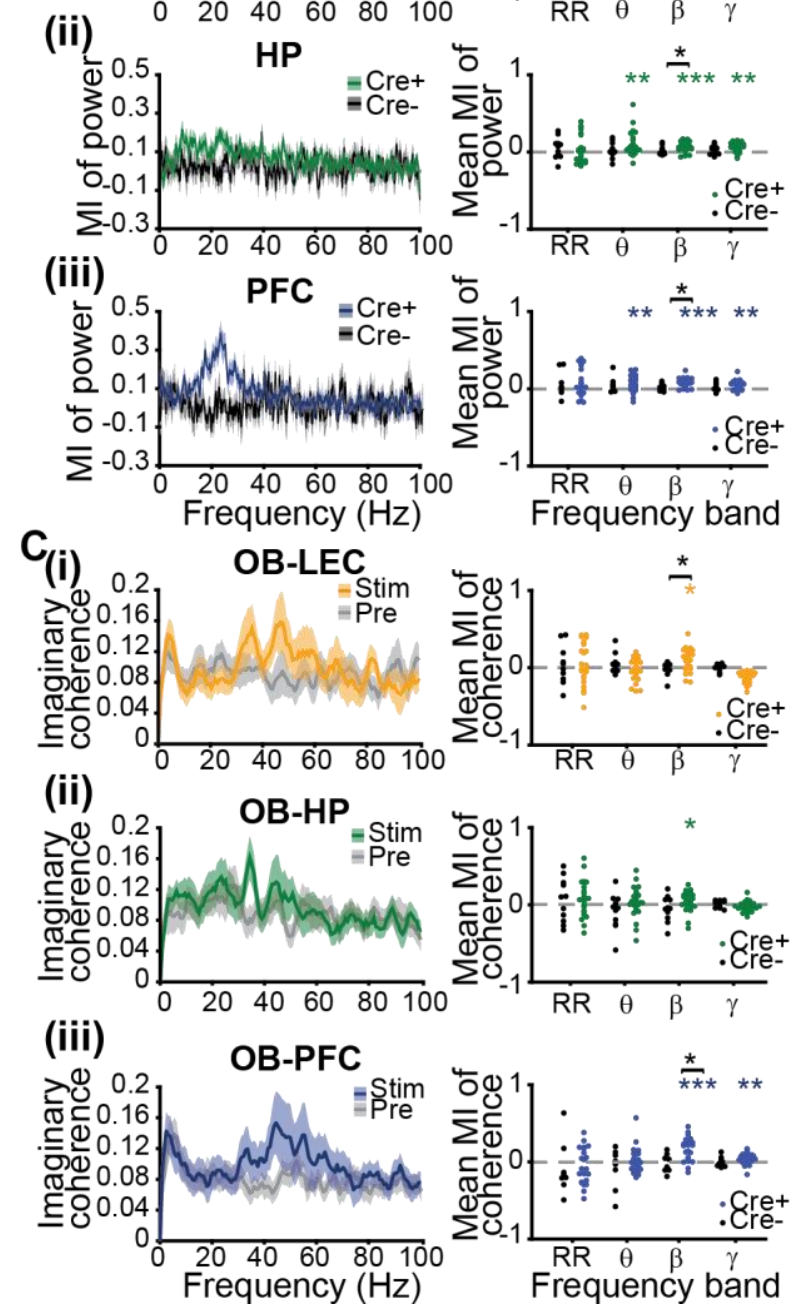

Fig 4. Oscillatory entrainment of limbic circuits as a result of $M / T C$ activation by light.

(A) Representative LFP traces recorded in the OB, LEC, HP, and PFC during ramp stimulation of ChR2-transfected M/TCs accompanied by the corresponding wavelet spectra. Vertical black lines correspond to $100 \mu \mathrm{V}$. (B) (i) Left, plot of MI for power during ramp stimulation of oscillations in LEC for $\mathrm{cre}^{+}$(yellow) and cre- (black) mice. Right, MI of LFP power averaged for different frequency bands for $\mathrm{cre}^{+}$(yellow) and cre- (black) mice. (ii) Same as (i) for HP. (iii) Same as (i) for PFC. (colored stars for $\mathrm{cre}^{+}$, gray stars for $\mathrm{cre}^{-},{ }^{*} \mathrm{p}<$ $0.05,{ }^{* *} p<0.01,{ }^{* * *} p<0.001$, Wilcoxon signedrank test; black stars for comparison cre+ vs. cre: ${ }^{*} p<0.05$, Wilcoxon rank-sum test). (C) (i) Left, imaginary coherence between OB and LEC before (gray) and during (yellow) light stimulation. Right, MI of LFP coherence averaged for different frequency bands for cre+ (yellow) and cre- (black) mice. (ii) Same as (i) for OB and HP. (iii) Same as (i) for OB and PFC. (colored stars for $\mathrm{cre}^{+:}{ }^{*} \mathrm{p}<$ 0.05, ${ }^{* *} p<0.01,{ }^{* * *} p<0.001$, Wilcoxon signedrank test; black stars for comparison cre $^{+}$vs. cre: ${ }^{*} p<0.05$, Wilcoxon rank-sum test). 
Inhibition of M/TC output reduces oscillatory power as well as neuronal firing in $\mathrm{OB}$,

\section{LEC, and HP}

To elucidate whether M/TC activity is necessary for the generation of oscillatory activity in downstream areas, we used inhibitory DREADDs (hM4D(Gi)) that block vesicle release when expressed in M/TCs by cre-dependent virus vector injection (AAV9-EF1a-DIO-hM4D(Gi)mCherry) at P1 (Fig 5A). At P8, M/TC soma as well as their axons forming the lateral olfactory tract (LOT), which targets the posterior part of the cerebrum, expressed hM4D(Gi)-mCherry (Fig 5B). We performed extracellular recordings of LFP and SUA from OB, LEC, and HP of P810 mice ( $n=35)$ before (baseline, $20 \mathrm{~min}$ ) and after (40 min) subcutaneous injection of C21 (3 $\mathrm{mg} / \mathrm{kg}$ ), a synthetic activator of DREADDs [42] (Fig 5A). Since the impact of OB activation on PFC was rather weak, we did not monitor its activity during OB silencing.

C21 caused broadband power reduction in OB that reached a maximum magnitude within 5 min after the injection (Figs 5C, 5D, and S7 Table) and persisted for at least $2 \mathrm{~h}$ (S3C Fig). The occurrence of discontinuous oscillatory events was lower after C21 injection in OB (Fig 5F and S8 Table), indicating that $\mathrm{M} / \mathrm{TC}$ activity is involved in the generation of discontinuous events in OB. Solely, the continuous $\mathrm{RR}$ in $\mathrm{OB}$ was not affected by the activation of DREADDs (Fig 5E and S7 Table). Moreover, silencing the M/TC output led to a broadband reduction of oscillatory power in LEC and HP (Figs 5C-E and Table S7). Correspondingly, the time spend in oscillatory events in LEC and HP decreased after inhibition of M/TC output (Fig 5F and S8 Table). In contrast, for cre mice LFP power and time spend in oscillatory events did not differ before and after C21 injection (S4A and S4B Figs, S7 and S8 Tables).

Next, we monitored the effects of chemogenetic silencing of M/TCs on the neuronal firing of downstream areas. Inhibitory DREADDs have been described to mainly reduce the vesicle release in the expressing neurons, while having little, if any, impact on their ability to generate action potentials $[43,44]$. Indeed, C21 injection had a weak effect on SUA in OB (cre+: med Ml: -0.071 , iqr: $-0.322-0.208, n=512, p=0.003$, Wilcoxon signed-rank test; cre : med Ml: -0.028 , iqr: $-0.259-0.207, n=418, p=0.171$, Wilcoxon signed-rank test; cre $^{+}$vs. cre $: p=0.198$, Wilcoxon rank-sum test) (Figs $5 \mathrm{G}$ and $5 \mathrm{H}$ ). In particular, the neuronal firing within the first 10 
342

343

344

345

min after C21 injection decreased (Fig 5G), being most likely the result of weaker network interactions within the OB. The DREADDs manipulation affected not only the network and neuronal activity in OB but also the spike timing by oscillations. In line with the results of spiketriggered power (STP) analysis, C21 injection decreased the ability of SUA to entrain the OB in theta, beta, and gamma rhythms (S4A Fig and S9 Table). STP for RR was comparable in the presence and absence of C21 (S9 Table). The temporal relationship between OB spikes and oscillatory events in OB was also assessed by calculating the phase-locking of SUA to RR and beta rhythm, respectively. In line with the results of STP analysis, the phase-locking to beta (Baseline: med: 0.105, iqr: $0.061-0.152 ; \mathrm{C} 21$ : med: 0.093 , iqr: $0.053-0.139 ; \mathrm{n}_{\text {units }}=524$ from 16 mice, $p=0.003$, LMEM) was reduced after C21 injection. In contrast, the phase coupling to RR (Baseline: med: 0.094, iqr: $0.051-0.153$; C21: med: 0.159, iqr: $0.078-0.310 ; \mathrm{n}_{\text {units }}=524$ from 16 mice, $p=2.20^{\star} 10^{-16}$, LMEM) was increased after C21 injection (S4B Fig).

Silencing the M/TC output strongly reduced the LEC firing (cre+: med MI: -0.420 , iqr: $0.598--0.108, n=126, p=2.96^{*} 10^{-16}$, Wilcoxon signed-rank test; cre: med MI: 0.069, iqr: $0.144-0.364, n=49, p=0.168$, Wilcoxon signed-rank test; cre $^{+}$vs. cre $: p=3.87^{*} 10^{-10}$, Wilcoxon rank-sum test), the effects lasting $>1$ hour after $\mathrm{C} 21$ injection (Figs $5 \mathrm{G}$ and $5 \mathrm{H}$ ). In contrast, silencing of M/TC output had a shorter ( 20 min) and weaker impact on hippocampal firing (cre+: med Ml: -0.102 , iqr: $-0.438-0.230, \mathrm{n}=102, \mathrm{p}=0.036$, Wilcoxon signed-rank test; cre: med MI: -0.047 , iqr: $-0.256-0.109, n=74, p=0.119$, Wilcoxon signed-rank test; cre $^{+}$vs. cre: $\mathrm{p}=0.484$, Wilcoxon rank-sum test).

These results indicate that silencing the M/TC output decouples neuronal firing from beta oscillations in $\mathrm{OB}$ and decreases the oscillatory power and neuronal firing in LEC, as a first downstream station of OB projections. On its turn, the weaker drive from LEC leads to poor oscillatory entrainment of HP, yet without significant change of its neuronal firing. 
A

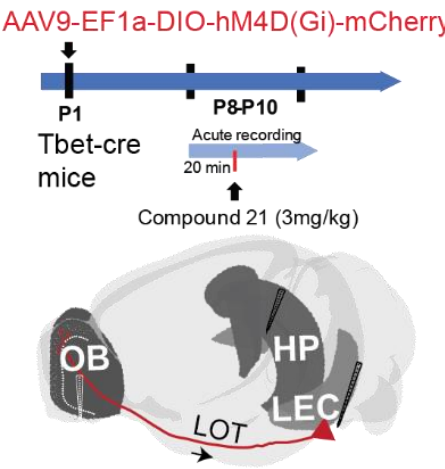

C
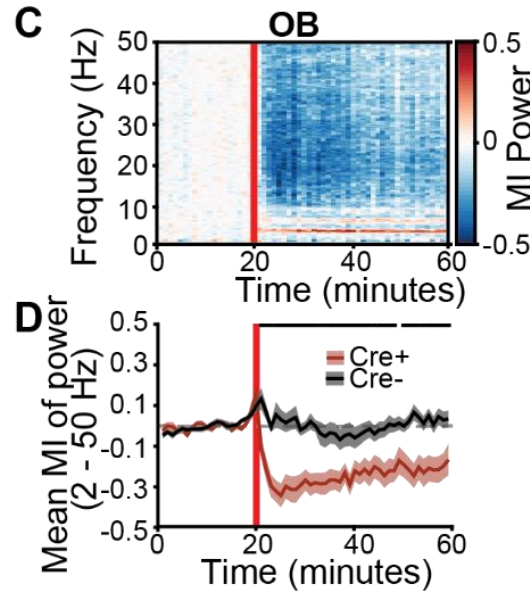

E

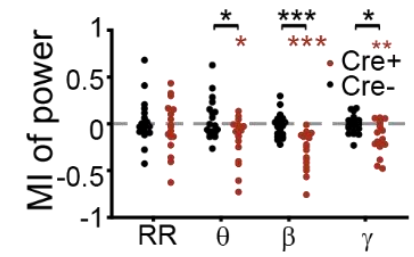

$\mathbf{F}$

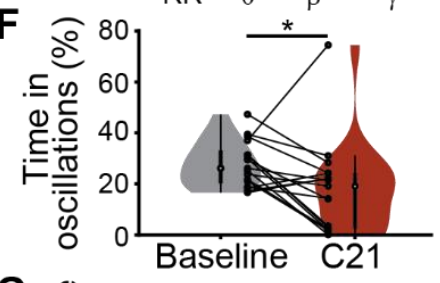

G
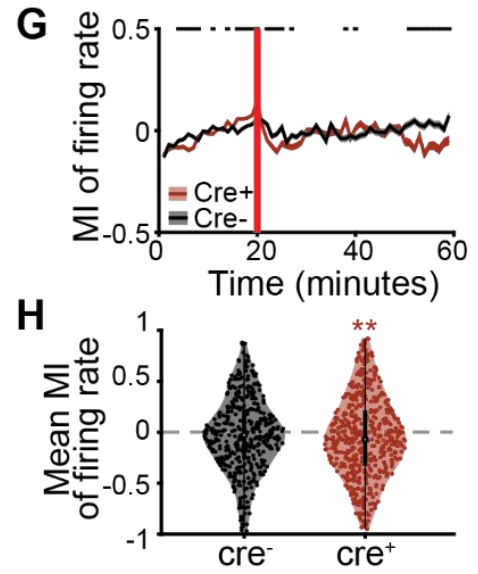
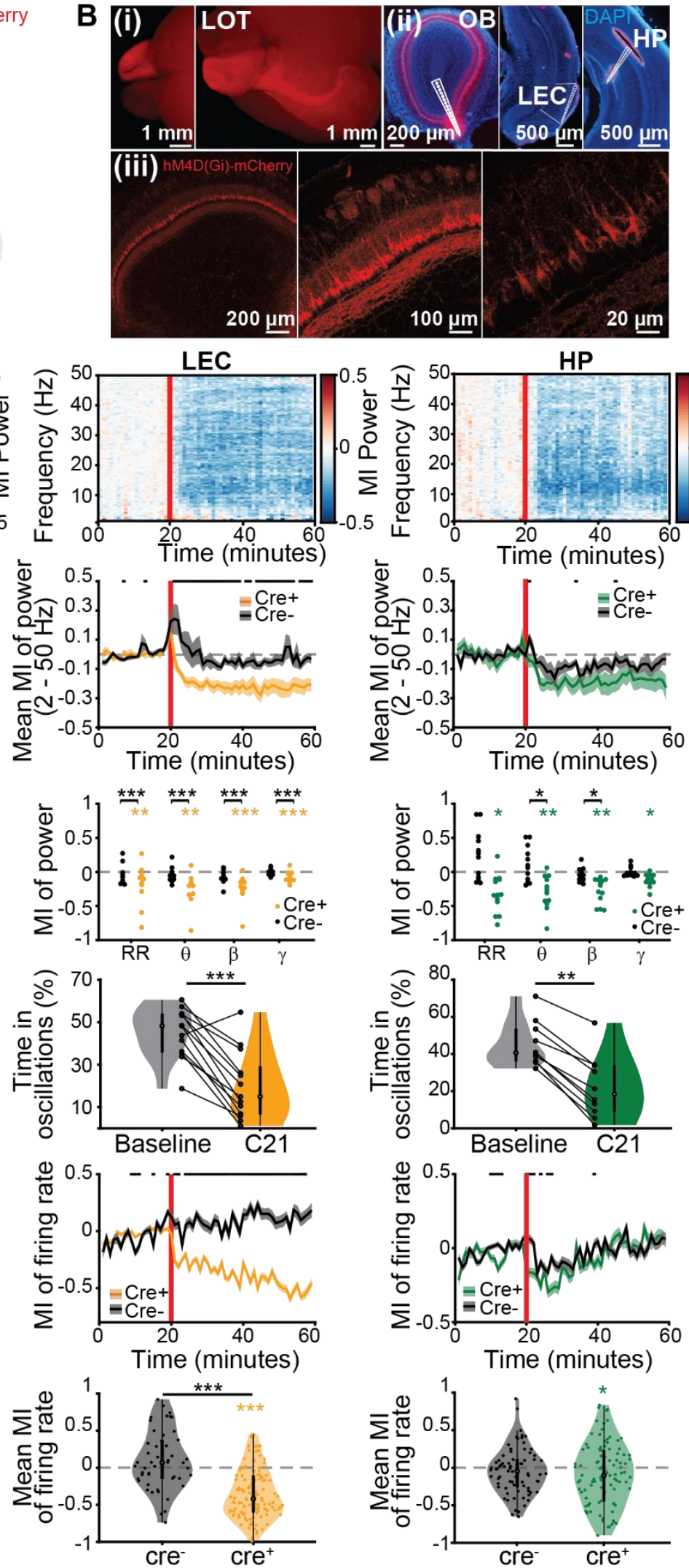

Fig 5. Effects of silencing M/TC output by inhibitory DREADDs on the oscillatory activity in $O B$, LEC, and HP. 
bioRxiv preprint doi: https://doi.org/10.1101/2021.10.04.463041; this version posted October 21, 2021. The copyright holder for this preprint (which was not certified by peer review) is the author/funder. All rights reserved. No reuse allowed without permission.

Fig 5. Effects of silencing M/TC output by inhibitory DREADDs on the oscillatory activity in OB, LEC, and HP.

(A) Top, schematic of the experimental protocol. Bottom, schematic of recording configuration for simultaneous extracellular recordings in OB, LEC, and HP (Brainrender: [39]). (B) (i) Photograph of the dorsal (left) and ventral side (right) of a brain from a P8 Tbet-cre+ mouse showing mCherry (red) expression in the $\mathrm{OB}$ and M/TC axonal projections (LOT) to PIR and LEC. (ii) Digital photomontages displaying the Dil labeled electrode track (red) in DAPI (blue) stained slices including the OB (left), LEC (middle), and HP (right) from a P10 mouse. (iii) Confocal images displaying the MCL of the right OB at different magnifications. MC bodies, as well as dendrites, express mCherry. (C) Color-coded MI of LFP power before and after C21 injection in OB (left), LEC (middle), and HP (right). Vertical red lines correspond to the $\mathrm{C} 21$ injection. (D) Plots displaying the MI of LFP power averaged for 2 to $50 \mathrm{~Hz}$ before and after C21 injection in cre+ (colored) and cre- (black) mice for OB (right, red), LEC (middle, yellow), and HP (right, green). Vertical red lines correspond to the C21 injection. (black line: $p<0.05$, Wilcoxon rank-sum test). (E) MI of LFP power averaged for different frequency bands for $\mathrm{cre}^{+}$(colored) and cre(black) mice for OB (left), LEC (middle), and HP (right). (Wilcoxon signed-rank test, colored stars for cre $^{+}:{ }^{*} p<0.05,{ }^{* *} p<0.01,{ }^{* * *} p<0.001$; black stars for comparison cre ${ }^{+}$vs. cre: : $p<0.05,{ }^{* *} p<0.01$, ${ }^{* * *} p<0.001$, Wilcoxon rank-sum test). (F) Violin plots displaying the percentage of time spend in discontinuous oscillatory events before (Baseline, gray) and after C21 injection (C21, colored). Black dots and lines correspond to individual animals. $\left({ }^{*} p<0.05\right.$, ${ }^{* *} p<0.01,{ }^{* * *} p<0.001$, Wilcoxon signedrank test). (G) Line plots displaying the MI of averaged SUA firing rates before and after C21 injection in cre $^{+}$(colored) and cre- (black) mice for OB (left, red), LEC (middle, yellow), and HP (right, green). Vertical red lines correspond to the C21 injection. (black line: $p<0.05$, Wilcoxon signed-rank test). $(\mathbf{H})$ Violin plots displaying the $\mathrm{Ml}$ of averaged SUA firing rates after $\mathrm{C} 21$ injection for cre- (black) and cre ${ }^{+}$ mice (colored) recorded in OB (left, red), LEC (middle, yellow), and HP (right, green). Red and black dots correspond to individual units. (colored stars for $\mathrm{cre}^{+:}$: $p<0.05$, ${ }^{* *} p<0.01$, ${ }^{* * *} p<0.001$, Wilcoxon signed-rank test; black stars for comparison cre ${ }^{+}$vs. cre: ${ }^{* * *} p<0.001$, Wilcoxon rank-sum test)

\section{Inhibition of M/TC output reduces the communication between $\mathrm{OB}$ and downstream}

\section{cortical areas}

To back up the hypothesis that the M/TC activity controls the developmental entrainment of limbic circuits, we monitored the communication between $\mathrm{OB}$ and downstream areas during silencing of M/TC output with DREADDs by using three distinct measures. First, we assessed the synchrony between $\mathrm{OB}, \mathrm{LEC}$, and HP by calculating the imaginary coherence in different frequency bands before (baseline) and after C21 injection (C21) (Figs 6A and 6B). Mls for beta coherence between $\mathrm{OB}$ and $\mathrm{LEC}$, and $\mathrm{OB}$ and $\mathrm{HP}$ were significantly reduced after $\mathrm{C} 21$ 
401

402

403

404

405

406

407

408

409

410

411

412

413

414

415

416

417

418

419

420

421

422

injection. In contrast, the coherence in other frequency bands was not affected by C21 injection (Figs 6A and 6B, S10 Table). Moreover, the C21-induced changes in the beta band were not detected in cre- mice (S6A Fig and S10 Table).

Second, we calculated the phase-amplitude coupling (PAC) to elucidate the role of M/TCs in the modulation of cortical beta oscillations by the RR phase in OB. C21 injection significantly reduced the z-scored PAC values between the OB RR phase and the amplitude of beta oscillations in LEC (Baseline: med: 2.499, iqr: $1.624-2.883$; C21: med: 1.608, iqr: $0.674-2.361, n=13, p=0.017$, Wilcoxon signed-rank test) and HP (Baseline: med: 2.363, iqr: 2.135-2.764; C21: med: 1.907 , iqr: $1.319-2.248, n=10, p=0.037$, Wilcoxon signed-rank test) (Fig 6C). Additionally, fewer mice showed significant RR-beta PAC values after C21 injection (z-score > 1.96) in LEC (Baseline: $53.85 \%$ vs. C21: $39.77 \%$ ) and HP (90\% vs. $50 \%$ ).

Third, we tested the effect of $\mathrm{C} 21$ on the directionality of interactions between OB, LEC, and HP (Fig 6D). We calculate the SDR and found that the prominent drive from OB to LEC was absent after silencing of M/TC output, the values for OB $\rightarrow$ LEC and LEC $\rightarrow$ OB being comparable (Fig 6Di, S11 and S12 Tables). Similarly, the drive from OB to HP was disrupted by C21 injection (Fig 6Diii, S11 and S12 Tables). In contrast, the directionality of interactions between LEC and HP was not affected by C21 injection. As reported for the baseline conditions, the mutual interactions LEC-HP persisted after M/TC silencing (Fig 6Dii, S11 and S12 Tables). Moreover, the C21-induced changes in directionality were not detected in cremice (S5B Fig, S11 and S12 Tables).

Thus, these results show that the $\mathrm{M} / \mathrm{TC}$ activity is critical for the communication between OB and its downstream cortical areas. 
A
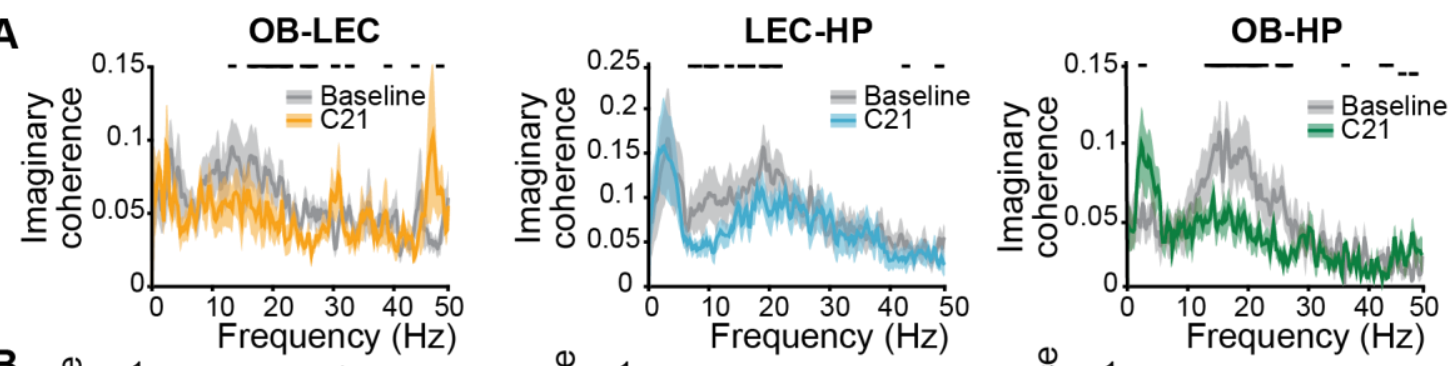

$B \quad 0$
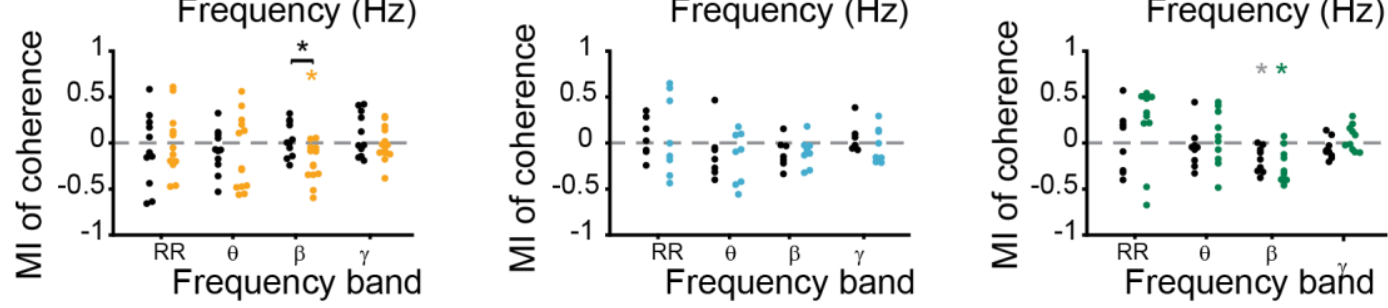

C(i)

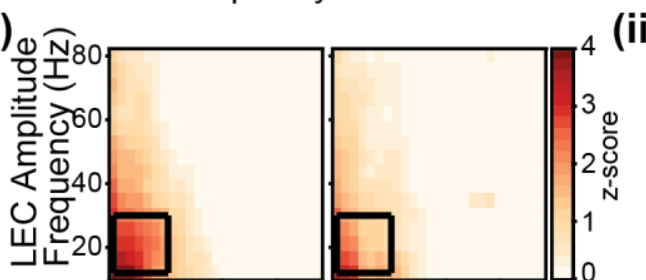

(ii)

Frequency band

(iii)
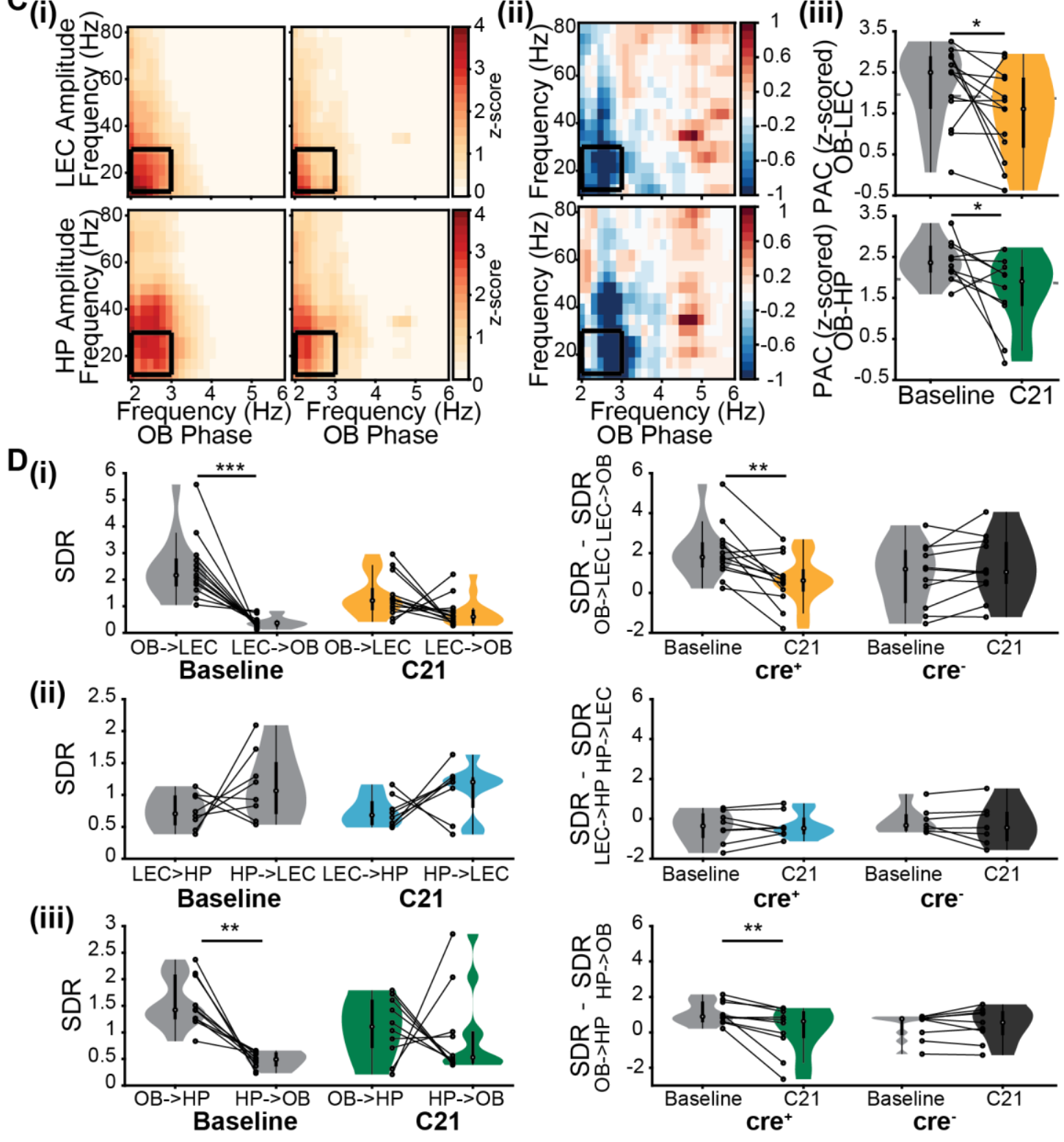

Fig 6. Modulation of functional communication within olfactory-cortical networks through silencing the M/TC output by inhibitory DREADDs. 
Fig 6. Modulation of functional communication within olfactory-cortical networks through silencing the M/TC output by inhibitory DREADDs.

(A) Imaginary coherence calculated for OB - LEC (left, yellow), LEC - HP (middle, light blue), and OB HP (right, green), before (Baseline, gray) and after C21 injection (C21, colored). (black line: $p<0.05$, Wilcoxon rank-sum test). (B) MI of coherence averaged for different frequency bands between $\mathrm{OB}$ and LEC (left, yellow), LEC and HP (middle, light blue), and OB and HP (right, green), for cre ${ }^{+}$(colored) and cre- (black) mice. (colored stars for cre $^{+}$, gray stars for cre: ${ }^{*} p<0.05$, Wilcoxon signed-rank test; black stars for comparison cre ${ }^{+}$vs. cre: : $p<0.05$, Wilcoxon rank-sum test). (C) (i) Z-scored phase-amplitude coupling (PAC) between OB phase and LEC (top) and HP (bottom) amplitude, before (Baseline) and after C21 injection (C21). (ii) Difference of PAC values after and before C21 injection for OB - LEC (top) and OB - HP (bottom). (iii) PAC averaged for RR-beta coupling (black box in (i)) for OB - LEC (top) and OB - HP (bottom), before (Baseline, gray) and after C21 injection (colored). Dotted gray line corresponds to a z-score of 1.96. ( ${ }^{*} p<0.05$, Wilcoxon signed-rank test). (D) (i) SDR calculated for OB and LEC. Left, SDR values for OB -> LEC and LEC -> OB before (Baseline, gray) and after C21 injection (C21, yellow). Right, difference of SDR values for both directions for $\mathrm{cre}^{+}$and cre- mice. (ii) Same as (i) for LEC and HP (blue). (iii) Same as (i) for OB and HP (green). Black dots and lines correspond to individual animals. $\left({ }^{* *} p<0.01,{ }^{* * *} p<0.001\right.$, Wilcoxon signed-rank test).

\section{DISCUSSION}

Long-range interactions within limbic circuits emerge early in life [45] yet it is still unknown whether the coordinated activity patterns underlying the coupling are endogenously generated or result through the driving force of sensory systems. Besides muscle twitches [46] and passive tactile sensation, olfactory inputs are likely candidates for the instruction of limbic circuitry development. Newborn rodents are not only able to smell from birth on but, importantly, also use olfactory information for learning and cue-directed behaviors such as localization of the nipples of the dam [20,47]. A first piece of evidence for the critical role of olfaction for limbic development is the fact that the neonatal OB shows functional coupling with the LEC, the gatekeeper of the limbic circuitry, during discontinuous network oscillations in the theta-beta frequency range as well as in the continuous respiration-related rhythm (RR) [34]. Here, we extended these findings and uncovered that MC firing sets a beta band entrainment also in downstream areas, such as HP and PFC. The temporal dynamics of oscillatory and 
firing activity revealed that even in periods without active odor sampling, OB drives the activation of limbic circuits.

Layer-specific analysis of SUA revealed that M/TC activation leads to a complex entrainment of the $\mathrm{OB}$ microcircuit that results in augmented firing rate also for interneurons in the GCL, EPL, and GL. Experimental and modeling studies have shown that both beta and gamma oscillations in the $\mathrm{OB}$ rely on dendro-dendritic interactions between M/TCs and GCs [24,48-51]. In adults, the emergence of gamma and beta oscillations is controlled by different excitability states of GCs as well as their dependency on centrifugal input, with beta oscillations relying on a higher GC excitability and centrifugal feedback projections $[30,48,50]$. However, gamma oscillations are absent in the neonatal $\mathrm{OB}$, most likely as a result of the late functional integration of interneurons into local circuits and the different biophysical properties of MCs and GCs during development [52-54]. Instead, discontinuous beta band oscillations are present not only in OB but also in other sensory and limbic areas [10]. In the neonatal PFC, they have been shown to accelerate along development until reaching the gamma band range at juvenile age [55]. Similarly, acceleration of beta to gamma oscillations takes place in V1 during the critical period for vision $[56,57]$. Whether the beta band activity in OB undergoes a similar transition to faster rhythms and how this process is controlled by interactions within $\mathrm{OB}$ and by feedback projections from PiR and LEC remain to be elucidated.

The present data show that the OB network activation entrains downstream cortical areas in beta oscillations. In adult rodents, the axonal terminals of MCs have been found to target fan and pyramidal neurons in LII/III of LEC that, on their turn, relay this information to the HP [58,59]. The axonal projections from layer II/III LEC pyramidal neurons to CA1 are involved in associative odor learning in adults [60]. Already at neonatal age, MC axons reach layer I of LEC $[34,61]$. Here, projections of layer II/III neurons that target the HP were detected and they might establish synaptic contacts with the MC axons. Optogenetic stimulation revealed that the activation of M/TCs induced delayed firing of LEC neurons and HP neurons, indicating that the pathway OB-to-HP is indeed already functional from birth on. CA1 receives entorhinal input not only via the direct performant path but also through the tri-synaptic path, 
480

481

482

483

484

485

486

487

488

489

490

491

492

493

spanning DG and CA3 [62]. The long latency ( $60 \mathrm{~ms})$ in light-induced CA1 firing might, therefore, be partly mediated by the tri-synaptic path. Of note, it was recently shown that a distinct but rather small population of LEC layer II neurons directly projects to the neonatal PFC [17], yet light stimulation of M/TCs did not recruit it.

Coordinated activity patterns in OB organized by MCs promote not only neuronal firing but also network activation in downstream areas. Ramp light stimulation of M/TCs led to an increase of beta band power in LEC, HP, and PFC. This power surge was accompanied by increased long-lasting SUA firing in all three brain areas, indicating that the initial activation of neurons is followed by activation of the local networks in LEC, HP, and PFC. Conversely, blocking vesicle release on MC synapses by DREADDs reduced the broadband power as well as neuronal firing in LEC and HP. Moreover, coherence analysis revealed increased oscillatory, mainly beta band coupling, between $\mathrm{OB}$ and cortical areas during ramp stimulation, whereas inhibition of M/TCs vesicle release reduced the drive $\mathrm{OB} \rightarrow \mathrm{LEC}$ and $\mathrm{OB} \rightarrow \mathrm{HP}$ as well as RR-beta cross-frequency coupling and beta coherence between OB-LEC and OB-HP. While the artificial activation of MCs might not be entirely comparable to the neural processes underlying odor sampling and processing during a learning task, these results identify the beta rhythm as a potential mechanism of long-range communication between $\mathrm{OB}$ and downstream cortical networks.

What might be the relevance of OB-controlled beta band activation of cortical circuits during early postnatal development? Beta oscillations have been reported to play a key role in working memory and decision making in adult humans [63]. Further, prominent beta band synchrony between cortical areas has been identified during olfactory-guided memory and decision making tasks in rodents $[29,31,33,64]$. A similar, but sniffing-independent increase in hippocampal beta oscillations has been observed during an object learning task [65]. Moreover, the firing of beta-entrained CA1 interneurons during an odor-place associative memory and decision-making task is related to an accurate performance, indicating that beta oscillations enable temporal coordination and recruitment of neurons within functional cell 
assemblies $[33,64]$. In line with these experimental data, modeling confirmed that beta oscillations optimally contribute to the coupling of cell assemblies over long axonal conductance delays [66-68]. During development, discontinuous beta band events that have been identified in PFC, HP, and LEC might facilitate the formation of initial cell assemblies with relevance for cognitive performance later in life. We previously showed that interfering with beta band oscillations during a defined developmental period causes network miswiring and poor behavioral performance of adult mice [19]. Similarly, in a mouse model of psychiatric risk reduced beta band activity at neonatal age has been found to correlate with later cognitive deficits $[13,17]$. Here, we identified the olfactory activity as a prominent driver of these early beta oscillations. The results let us hypothesize that transient disturbance of neonatal olfactory processing precludes the functional refinement of entorhinal-hippocampal-prefrontal circuits, ultimately leading to cognitive deficits in adulthood. Further research is warranted to directly test this hypothesis and elucidate the role of early activity patterns in $O B$ for cognitive development.

\section{MATERIALS AND METHODS}

\section{Ethical Approval}

All experiments were performed in compliance with the German laws and the guidelines of the European Union for the use of animals in research (European Union Directive 2010/63/EU) and were approved by the local ethical committee (Behörde für Gesundheit und Verbraucherschutz Hamburg, ID 15/17).

\section{Animals}

Time-pregnant C57BI/6/J and Tbet-cre mice from the animal facility of the University Medical Center Hamburg-Eppendorf were housed individually in breeding cages at a $12 \mathrm{~h}$ light / $12 \mathrm{~h}$ dark cycle and fed ad libitum. Offspring (both sexes) where injected with either AAV9-Ef1aDIO-hChR2(E123T_T159C)-EYFP (Addgene, Plasmid \#35509) or AAV9-EF1a-DIOhM4D(Gi)-mCherry (Addgene, Plasmid \#50461) virus at postnatal day (P) 0 or 1 . Genotypes 
534

535

536

537

538

539

540

541

542

543

were determined using genomic DNA and following primer sequences (Metabion, Planegg/Steinkirchen, Germany) as described previously [34]: for Cre: PCR forward primer 5'ATCCGAAAAGAAAACGTTGA-3' and reverse primer 5'-ATCCAGGTTACGGATATAGT-3'. The PCR reactions were as follows: $10 \mathrm{~min}$ at $95^{\circ} \mathrm{C}, 30$ cycles of $45 \mathrm{~s}$ at $95^{\circ} \mathrm{C}, 90 \mathrm{~s}$ at $54^{\circ} \mathrm{C}$, and $90 \mathrm{~s}$ at $72{ }^{\circ} \mathrm{C}$, followed by a final extension step of $10 \mathrm{~min}$ at $72{ }^{\circ} \mathrm{C}$. In addition to genotyping, EGFP expression in OB was detected using a dual fluorescent protein flashlight (Electron microscopy sciences, Hatfield, PA, USA) prior to surgery. At P8-10 cre- and cre ${ }^{+}$mice underwent light stimulation or Compound 21 injections and in vivo multi-side electrophysiological recordings.

\section{Surgical procedures and recordings}

Virus injection for transfection of MTCs with ChR2 and hm4D(Gi)

For transfection of $\mathrm{M} / \mathrm{TCs}$ with the ChR2 derivate $\mathrm{E} 123 \mathrm{~T} / \mathrm{T} 159 \mathrm{C}$ or inhibitory DREADDs $(\mathrm{hm} 4 \mathrm{D}(\mathrm{Gi})), \mathrm{P} 0-1$ pups were fixed into a stereotaxic apparatus and received unilateral injections of one of two viral constructs (AAV9-Ef1a-DIO hChR2(E123T/T159C)-EYFP, $200 \mu \mathrm{l}$ at titer $\geq 1 \times 10^{13} \mathrm{vg} / \mathrm{mL}$, Plasmid, \#35509, Addgene, MA, USA; AAV9-EF1a-DIO-hM4D(Gi)mCherry, $200 \mu \mathrm{l}$ at titer $\geq 1 \times 10^{14} \mathrm{vg} / \mathrm{mL}$ Plasmid \#50461, Addgene, MA, USA). The virus was produced by Addgene or the Virus Facility of the University Medical Center Eppendorf. A total volume of $200 \mathrm{nl}$ was slowly (200 $\mathrm{nl} / \mathrm{min}$ ) delivered at a depth of around $0.5 \mathrm{~mm}$ into the right OB using a micropump (Micro4, WPI, Sarasota, FL). Following injection, the syringe was left in place for at least $30 \mathrm{~s}$ to avoid reflux of fluid. Pups were maintained on a heating blanket until full recovery and returned to the dam.

\section{Virus injection for tracing}

For the transfection of M/TC axons with EYFP and the retrograde labeling of HP-projecting neurons with mCherry, P0-1 pups received the viral construct AAV9-hSyn-hChR2(H134R)EYFP $\left(200 \mu \mathrm{l}\right.$ at titer $\geq 1 \times 10^{13} \mathrm{vg} / \mathrm{mL}$, \#26973-AAV9, Addgene, MA, USA) into the OB and the retrograde virus AAVrg-CamKIl $\alpha$-mCherry $\left(80 \mu \mathrm{l}\right.$ at titer $\geq 7 \times 10^{12} \mathrm{vg} / \mathrm{mL}$, \#114469-AAVrg, Addgene, MA, USA) into the HP. Virus injection was performed similarly as for the transfection 
561

562

563

564

565

566

567

568

569

570

571

572

573

574

575

576

577

578

579

580

581

582

583

584

585

586

587

of $\mathrm{M} / \mathrm{TC}$ with $\mathrm{ChR} 2$ or hm4D(Gi). After 10 days, the brains of investigated mice were perfused with 4\% paraformaldehyde (PFA), sliced and MC axons and HP-projecting neurons in LEC and PIR were imaged using a confocal microscope.

\section{Surgical procedure for electrophysiology}

For in vivo recordings, P8-10 mice underwent surgery according to previously described protocols $[11,34,35]$. Under isoflurane anesthesia (induction: $5 \%$, maintenance: $2.5 \%$, Forane, Abbott), the skin above the skull was removed and $0.5 \%$ bupivacaine / $1 \%$ lidocaine was locally applied on the neck muscles. Two plastic bars were mounted on the nasal and occipital bones with dental cement. The bone above the right $\mathrm{OB}(0.5-0.8 \mathrm{~mm}$ anterior to frontonasal suture, $0.5 \mathrm{~mm}$ lateral to inter-nasal suture), LEC ( $0 \mathrm{~mm}$ posterior to lambda, 6-7.5 $\mathrm{mm}$ lateral from the midline), HP (2.5 mm anterior to lambda, $3.5 \mathrm{~mm}$ lateral from the midline) and PFC (0.5 mm anterior to bregma, 0.1-0.5 mm lateral from the midline) was carefully removed by drilling a hole of $<0.5 \mathrm{~mm}$ in diameter. Throughout surgery and recording session the mice were maintained on a heating blanket at $37^{\circ} \mathrm{C}$.

\section{Multi-site electrophysiological recordings in vivo}

Three-side or four-side recordings were performed in non-anesthetized P8-10 mice. For this, one-shank electrodes (NeuroNexus, MI, USA) with 16 recording sites (0.4-0.8 M $\Omega$ impedance, $50 \mu \mathrm{m}$ inter-site spacing for recordings in $\mathrm{OB}$ and $\mathrm{HP}, 100 \mu \mathrm{m}$ inter-site spacing for recordings in LEC and PFC) were inserted into $\mathrm{OB}\left(0.5-1.8 \mathrm{~mm}\right.$, angle $\left.0^{\circ}\right)$, LEC (for 4-side recordings, depth: $2 \mathrm{~mm}$, angle: $180^{\circ}$; for 3 -side recordings, depth: $2-2.5 \mathrm{~mm}$, angle: $\left.10^{\circ}\right)$, $\mathrm{HP}(1.3-1.9 \mathrm{~mm}$, angle $20^{\circ}$ ) and PFC (1.8-2.1 $\mathrm{mm}$, angle $0^{\circ}$ ). For light stimulation one-shank optrodes (NeuroNexus, MI, USA) with the same configuration as the electrodes were inserted in the OB. Before insertion, the electrodes were covered with Dil (1,1'-Dioctadecyl-3,3,3',3'tetramethylindocarbocyanine perchlorate, Molecular Probes, Eugene, OR). A silver wire was inserted into the cerebellum and served as a ground and reference electrode. Before data acquisition, a recovery period of 20 min following the insertion of electrodes was provided. Extracellular signals were band-pass filtered $(0.1 \mathrm{~Hz}-9 \mathrm{kHz})$ and digitized $(32 \mathrm{kHz}$ or 32,556 
588

589

590

591

592

593

594

595

596

597

598

599

600

601

602

603

604

605

606

607

608

609

610

611

612

613

$\mathrm{kHz}$ ) by a multichannel amplifier (Digital Lynx SX; Neuralynx, Bozeman, MO; USA) and Cheetah acquisition software (Neuralynx). Spontaneous activity was recorded for at least 20 min before light stimulation or Compound 21 (C21, Hellobio, Ireland) injection. The position of recording electrodes in OB, LEC, HP, and PFC was confirmed after histological assessment post-mortem. For the analysis of LFP in OB, the recording site centered in the EPL was used, whereas for HP the recording site located in the CA1 was considered. For analysis of LFP in LEC only recording sites that were histologically confirmed to be located in superficial entorhinal layers were used. Similarly, only recordings sites confined to the prelimbic subdivision of PFC were considered. For the analysis of spiking activity, all recording sites confirmed to be located in the areas of interest (OB, LEC, HP, and PFC) were considered. When necessary, spikes recorded in $\mathrm{OB}$ were assigned to specific layers according to the location of recording sites.

\section{Morphology}

Mice were anesthetized with 10\% ketamine (Ketamidor, Richter Pharma AG, Germany) / 2\% xylazine (Rompun, Bayer, Germany) in $0.9 \% \mathrm{NaCl}$ solution $(10 \mu \mathrm{g} / \mathrm{g}$ body weight, i.p.) and transcardially perfused with Histofix (Carl Roth, Germany) containing 4\% PFA. Brains were postfixed in 4\% PFA for $24 \mathrm{~h}$ and sliced. Slices (100 $\mu \mathrm{m}$-thick) were mounted with Fluoromount containing DAPI (Sigma-Aldrich, MI, USA). The positions of the Dil-labeled extracellular electrodes in the OB, LEC, HP, and PFC were reconstructed to confirm their location. Virus expression was verified by EYFP (for ChR2) or mCherry (for hM4D(Gi)) fluorescence in the right OB. For confocal imaging of EYFP or mCherry fluorescence in M/TCs, HP, and LEC, 50 $\mu \mathrm{m}$-thick slices mounted with Vectashield (CA, USA) were used.

\section{Light stimulation}

Activation of M/TCs was achieved by either ramp or pulse light stimulation applied using a diode laser ( $473 \mathrm{~nm}$; Omicron, Austria) which was controlled by an arduino uno (Arduino, Italy). For ramp stimulation, a light stimulus with linear increasing power ( $3 \mathrm{~s}$ rise time) was presented 
614

615

616

617

618

619

620

621

622

623

624

625

626

627

628

629

630

631

632

633

634

635

636

637

30-60 times. For pulse stimulation $3 \mathrm{~ms}$ light pulses at $2 \mathrm{~Hz}$ were delivered. Laser power was adjusted for every recording (1.37-5.15 $\mathrm{mW})$ to reliably induce neuronal firing.

\section{Compound 21 injection}

Compound 21 (3 mg/kg solved in $0.9 \% \mathrm{NaCl}$ ) was injected subcutaneously after $>20 \mathrm{~min}$ recording of baseline activity, while the mouse was fixed in the stereotaxic apparatus. The activity was recorded for $40-120$ min post-injection.

\section{Data Analysis}

LFP analysis. Data were analyzed offline using custom-written scripts in the MATLAB environment (MathWorks, Natick, MA). Data were first low-passed filtered $(<100 \mathrm{~Hz})$ using a third-order Butterworth filter before down-sampling by factor 20 to $1.6 \mathrm{kHz}$ to analyze LFP. All filtering procedures were performed in a manner preserving phase information.

Detection of oscillatory activity. Discontinuous network oscillations in the LFP recorded from OB, LEC, and HP before and after C21 injection were detected using a previously developed unsupervised algorithm [69]. Briefly, deflections of the root mean square of band-pass filtered $(4-100 \mathrm{~Hz})$ signals exceeding a variance-depending threshold (2 times the standard deviation from the mean) were assigned as oscillatory periods. Only oscillatory periods lasting at least 1 $\mathrm{s}$ were considered for analysis.

Power spectral density. Power spectral density was analyzed for either the entire baseline period, $2 \mathrm{~s}$ long periods before (Pre), and during light ramp stimulation (Stim) for recordings combined with optogenetic manipulation. For recordings paired with DREADD manipulation, the power was either calculated for every minute or averaged for the entire baseline period (19 min) and post C21 injection period (30 min). Power was calculated using Welch's method with non-overlapping windows of $2 \mathrm{~s}$ (ramp periods) or $3 \mathrm{~s}$ length. Time-frequency plots of power were calculated with a continuous wavelet transform (Morlet wavelet). 
638

639

640

641

642

643

644

645

646

647

648

649

650

651

652

653

654

655

656

657

Coherence. The imaginary part of coherence, which is insensitive to volume-conduction-based effects [41], was calculated for the same time periods as the power by taking the absolute value of the imaginary component of the normalized cross-spectrum:

$$
C_{X Y}(f)=\left|\operatorname{Im}\left(\frac{P_{X Y}(f)}{\sqrt{P_{X X}(f) P_{Y Y}(f)}}\right)\right| .
$$

Spectral Dependency Ratio. The Spectral Dependency Ratio (SDR) was calculated according to Shajarisales et al. [38] from the power spectral densities $\left(S_{x}(f)\right.$ and $\left.S_{y}(f)\right)$ of the signals $X$ and $Y$ :

$$
\begin{gathered}
S D R_{X \rightarrow Y}=\frac{\operatorname{mean}\left(S_{y}(f)\right)}{\operatorname{mean}\left(S_{x}(f)\right) * \text { mean }\left(\frac{S_{y}(f)}{S_{x}(f)}\right)} \\
S D R_{Y \rightarrow X}=\frac{\operatorname{mean}\left(S_{x}(f)\right)}{\operatorname{mean}\left(S_{y}(f)\right) * \text { mean }\left(\frac{S_{x}(f)}{S_{y}(f)}\right)}
\end{gathered}
$$

The most likely direction of causation is the one having significantly larger SDR values. (https://github.com/OpatzLab/HanganuOpatzToolbox/tree/master/LFP analysis/getSDR.m)

Spiking analysis. Single units were automatically detected and clustered using the pythonbased software klusta [70] and manually curated using phy (https://github.com/cortex-lab/phy).

The firing rate was computed by dividing the total number of spikes by the duration of the analyzed time window. To assess the spike probability, histograms of spike count using $1 \mathrm{~ms}$ bins were calculated for periods around the light pulse (50 ms before to $150 \mathrm{~ms}$ after) and normalized to the number of delivered light pulses. Cross-covariance of spike trains was calculated as described previously [34,36]. Briefly, cross-covariance for two spike trains $N_{i}$ and $N_{j}$, was estimated from the cross-correlation histogram $\left(U_{i j}^{T, b}(u)\right)$ as follows:

$$
\hat{q}_{i j}(u)=\frac{J_{i j}^{T, b}(u)}{b T}-\hat{P}_{i} \hat{P}_{j},
$$


658

659

660

661

662

663

664

665

666

667

668

669

670

671

672

673

674

675

676

677

678

679

680

( $b=$ binsize, $T$ observation period, $\left.\hat{P}_{i}=\frac{N_{i}(T)}{T}, \hat{P}_{j}=\frac{N_{j}(T)}{T}\right)$. The standardized cross-covariance was calculated as

$$
Q_{i j}(u)=\sqrt{\frac{b T}{P_{i} P_{j}}} \hat{q}_{i j}(u)
$$

with $P_{i}, P_{j}$ being the mean firing rates. Only pairs of units with firing rates $>0.05 \mathrm{~Hz}$ and significant standardized cross variance were considered. The Null hypothesis was rejected when $\left|Q_{i j}(u)\right|>Z_{\alpha} \cdot\left(Z_{\alpha}=\sqrt{2} \operatorname{erf}^{-1}\left(\frac{1-\alpha}{N_{\text {lags }}}\right) ;\right.$ two-tailed critical $z$ value at level $\left.\alpha=0.01\right)$. The standardized mean cross-variance for one unit was calculated as

$$
Q_{i}(u)=\sqrt{\frac{1}{K}} \sum_{j=1}^{K} Q_{i j}(u),
$$

(K=number of units in 2. Region) and the mean for all unit pairs as: $\left\langle Q_{i}(u)\right\rangle=\frac{1}{L^{2}} \sum_{i=1}^{L} Q_{i}(u)$.

Modulation index. The modulation index (MI) of power, coherence, firing rate, and STP for light stimulation or DREADD manipulation was calculated as

$$
M I=\frac{\text { Value }_{\text {Stim }}-\text { Value }_{\text {Pre }}}{\text { Value }_{\text {Stim }}+\text { Value }_{\text {Pre }}} .
$$

Spike-LFP coupling. Phase locking of spiking units to network oscillations was assessed using a previously described algorithm [36]. For this, the LFP signal was bandpass filtered $(2-4 \mathrm{~Hz}$ (RR), 4-12 Hz (theta), $12-30 \mathrm{~Hz}$ (beta), 30-100 Hz (gamma)) using a third-order Butterworth filter. The instantaneous phase was extracted using the Hilbert transform on the filtered signal. The coupling between spikes and network oscillations was tested for significance using the Rayleigh test for non-uniformity. For analysis of baseline properties (Fig 1) only neurons that showed significant phase locking were considered for the analysis of the mean phase angle and the locking strength, which was calculated as mean resulting vector length (RVL). For paired comparison of RVLs (Fig 2, S1, S4) all units with a firing rate higher than $0.01 \mathrm{~Hz}$ during baseline (18 $\mathrm{min}$ ) and after C21 injection (18 min, DREADD manipulation) or more than 10 spikes before (Pre) and during (Stim) light ramp pulses were considered. 
681

682

683

684

685

686

687

688

689

690

691

692

693

694

695

696

697

698

699

700

701

702

703

704

705

(https://github.com/OpatzLab/HanganuOpatzToolbox/blob/master/Spikes-LFP

analysis/getPPC_PLV.m)

Spike-triggered power. Spike-triggered power (STP) was calculated for the same time periods as RVL by taking the mean of the LFP power for $0.4 \mathrm{~s}$ long time windows centered on each spike.

Phase-amplitude coupling. Phase-amplitude coupling (PAC) between RR phase in OB and beta band amplitude in LEC and HP was calculated as previously described [71]. Briefly, the LFP signals were bandpass filtered and the Hilbert transform was used to extract the phase and amplitude, respectively. Subsequently, the amplitude of the beta-filtered signal in LEC or HP was determined at each phase of the filtered OB signal. The phase was divided into 16 bins and the mean amplitude for each bin was calculated and normalized to the total number of bins. The normalized modulation index (MI) was calculated as the deviation between an empirical and uniform amplitude distribution. MI matrices were $z$-scored and the average was calculated for $\mathrm{RR}(2-3 \mathrm{~Hz})$ - beta $(12-30 \mathrm{~Hz})$ coupling.

\section{Statistics}

Statistical analysis was performed in MATLAB environment or R Statistical Software. As none of the data sets were normally distributed, data were tested for significance using Wilcoxon rank-sum test (2 unrelated samples) or Wilcoxon sign-rank test (2 related samples). Data (except phase values) are presented as median (med) and interquartile range (iqr). Outlier removal was applied to paired data points if the distance of their difference from the $25^{\text {th }}$ or $75^{\text {th }}$ percentile exceeds 2.5 times the interquartile interval of their difference. Phase locking was tested for significance using the Rayleigh test for non-uniformity. Phase angles were compared using a circular non-parametric multi-sample test for equal medians. Differences in proportions were tested using Fisher's exact test. Nested data were analyzed with linear mixed-effects models (LMEM) using animals as a fixed effect. Significance levels ${ }^{*} \mathrm{p}<0.05,{ }^{* *} \mathrm{p}<0.01$ and ${ }^{* * *} \mathrm{p}<0.001$ were considered. If not included in the text, values and corresponding test statistics of all presented data can be found in the supplementary material (Table S1-12). 


\section{Additional Information}

709

710

711

712

713

714

715

\section{Acknowledgments}

We thank A. Marquardt, A. Dahlmann, P. Putthoff and K. Titze for excellent technical assistance, Dr. I. Braren from the Vector Facility of the UKE for the virus production as well as Drs. M. Chini, S.H. Bitzenhofer, and R.L. van den Brink for helpful discussions.

\section{Funding}

This work was funded by grants of the German Research Foundation (Ha4466/11-1 and SFB 936 B5 to I.L.H.-O), European Research Council (ERC-2015-CoG 681577 to I.L.H.-O.), Horizon 2020 DEEPER (101016787 to I.L.H.-O.), and Landesforschungsförderung Hamburg (LFF73 and LFF76 to I.L. H.-O.).

\section{Author Contributions}

I.L.H.-O. and J.K.K conceived the study and designed the experiments. J.K.K carried out the experiments and analyzed the data. J.K.K, and I.L.H.-O. interpreted the data. J.K.K. and I.L.H.O. wrote the article. All authors discussed and commented on the manuscript.

\section{Declaration of Interests}

The authors declare no competing interests. 
bioRxiv preprint doi: https://doi.org/10.1101/2021.10.04.463041; this version posted October 21,2021 . The copyright holder for this preprint (which was not certified by peer review) is the author/funder. All rights reserved. No reuse allowed without permission.

\section{REFERENCES}

1. Hanganu-Opatz IL. Between molecules and experience: Role of early patterns of coordinated activity for the development of cortical maps and sensory abilities. Brain Res Rev. 2010;64: 160-176. doi:10.1016/j.brainresrev.2010.03.005

2. Leighton AH, Lohmann C. The Wiring of Developing Sensory Circuits-From Patterned Spontaneous Activity to Synaptic Plasticity Mechanisms. Front Neural Circuits. 2016;10. doi:10.3389/fncir.2016.00071

3. Ackman JB, Burbridge TJ, Crair MC. Retinal waves coordinate patterned activity throughout the developing visual system. Nature. 2012;490: 219-225. doi:10.1038/nature11529

4. Hanganu IL, Ben-Ari Y, Khazipov R. Retinal Waves Trigger Spindle Bursts in the Neonatal Rat Visual Cortex. J Neurosci. 2006;26: 6728-6736. doi:10.1523/JNEUROSCI.075206.2006

5. Khazipov R, Sirota A, Leinekugel X, Holmes GL, Ben-Ari Y, Buzsáki G. Early motor activity drives spindle bursts in the developing somatosensory cortex. Nature. 2004;432: 758-761. doi:10.1038/nature03132

6. Mizuno H, Luo W, Tarusawa E, Saito YM, Sato T, Yoshimura Y, et al. NMDAR-regulated dynamics of layer 4 neuronal dendrites during thalamocortical reorganization in neonates. Neuron. 2014;82: 365-379. doi:10.1016/j.neuron.2014.02.026

7. Wang HC, Bergles DE. Spontaneous activity in the developing auditory system. Cell Tissue Res. 2015;361: 65-75. doi:10.1007/s00441-014-2007-5

8. Che A, Babij R, lannone AF, Fetcho RN, Ferrer M, Liston C, et al. Layer I Interneurons Sharpen Sensory Maps during Neonatal Development. Neuron. 2018;99: 98-116.e7. doi:10.1016/j.neuron.2018.06.002

9. Ahlbeck J, Song L, Chini M, Bitzenhofer SH, Hanganu-Opatz IL. Glutamatergic drive along the septo-temporal axis of hippocampus boosts prelimbic oscillations in the neonatal mouse. Bartos M, editor. eLife. 2018;7: e33158. doi:10.7554/eLife.33158

10. Bitzenhofer SH, Ahlbeck J, Wolff A, Wiegert JS, Gee CE, Oertner TG, et al. Layer-specific optogenetic activation of pyramidal neurons causes beta-gamma entrainment of neonatal networks. Nat Commun. 2017;8: 14563. doi:10.1038/ncomms14563

11. Brockmann MD, Pöschel B, Cichon N, Hanganu-Opatz IL. Coupled Oscillations Mediate Directed Interactions between Prefrontal Cortex and Hippocampus of the Neonatal Rat. Neuron. 2011;71: 332-347. doi:10.1016/j.neuron.2011.05.041

12. Hartung $\mathrm{H}$, Brockmann MD, Pöschel B, Feo VD, Hanganu-Opatz IL. Thalamic and Entorhinal Network Activity Differently Modulates the Functional Development of Prefrontal-Hippocampal Interactions. J Neurosci. 2016;36: 3676-3690. doi:10.1523/JNEUROSCI.3232-15.2016

13. Chini M, Pöpplau JA, Lindemann C, Carol-Perdiguer L, Hnida M, Oberländer V, et al. Resolving and Rescuing Developmental Miswiring in a Mouse Model of Cognitive Impairment. Neuron. 2020;105: 60-74.e7. doi:10.1016/j.neuron.2019.09.042

14. Domnick N-K, Gretenkord S, De Feo V, Sedlacik J, Brockmann MD, Hanganu-Opatz IL. Neonatal hypoxia-ischemia impairs juvenile recognition memory by disrupting the 
bioRxiv preprint doi: https://doi.org/10.1101/2021.10.04.463041; this version posted October 21,2021 . The copyright holder for this preprint (which was not certified by peer review) is the author/funder. All rights reserved. No reuse allowed without permission.

maturation of prefrontal-hippocampal networks. Exp Neurol. 2015;273: 202-214. doi:10.1016/j.expneurol.2015.08.017

15. Hartung $\mathrm{H}$, Cichon $\mathrm{N}$, De Feo V, Riemann $\mathrm{S}$, Schildt $\mathrm{S}$, Lindemann $\mathrm{C}$, et al. From Shortage to Surge: A Developmental Switch in Hippocampal-Prefrontal Coupling in a Gene-Environment Model of Neuropsychiatric Disorders. Cereb Cortex. 2016;26: 42654281. doi:10.1093/cercor/bhw274

16. Richter M, Murtaza N, Scharrenberg R, White SH, Johanns O, Walker S, et al. Altered TAOK2 activity causes autism-related neurodevelopmental and cognitive abnormalities through RhoA signaling. Mol Psychiatry. 2019;24: 1329-1350. doi:10.1038/s41380-0180025-5

17. Xu X, Song L, Kringel R, Hanganu-Opatz IL. Developmental decrease of entorhinal gate disrupts prefrontal-hippocampal communication in immune-challenged DISC1 knockdown mice. Res Sq. 2021. doi:10.21203/rs.3.rs-290304/v1

18. Krüger H-S, Brockmann MD, Salamon J, Ittrich H, Hanganu-Opatz IL. Neonatal hippocampal lesion alters the functional maturation of the prefrontal cortex and the early cognitive development in pre-juvenile rats. Neurobiol Learn Mem. 2012;97: 470-481. doi:10.1016/j.nlm.2012.04.001

19. Bitzenhofer SH, Pöpplau JA, Chini M, Marquardt A, Hanganu-Opatz IL. A transient developmental increase in prefrontal activity alters network maturation and causes cognitive dysfunction in adult mice. Neuron. 2021;109: 1350-1364.e6. doi:10.1016/j.neuron.2021.02.011

20. Logan DW, Brunet LJ, Webb WR, Cutforth T, Ngai J, Stowers L. Learned recognition of maternal signature odors mediates the first suckling episode in mice. Curr Biol CB. 2012;22: 1998-2007. doi:10.1016/j.cub.2012.08.041

21. Igarashi KM, leki N, An M, Yamaguchi Y, Nagayama S, Kobayakawa K, et al. Parallel Mitral and Tufted Cell Pathways Route Distinct Odor Information to Different Targets in the Olfactory Cortex. J Neurosci. 2012;32: 7970-7985. doi:10.1523/JNEUROSCI.015412.2012

22. Luskin MB, Price JL. The topographic organization of associational fibers of the olfactory system in the rat, including centrifugal fibers to the olfactory bulb. J Comp Neurol. 1983;216: 264-291. doi:10.1002/cne.902160305

23. Kay LM. Chapter 9 - Circuit Oscillations in Odor Perception and Memory. In: Barkai E, Wilson DA, editors. Progress in Brain Research. Elsevier; 2014. pp. 223-251. doi:10.1016/B978-0-444-63350-7.00009-7

24. Neville KR, Haberly LB. Beta and Gamma Oscillations in the Olfactory System of the Urethane-Anesthetized Rat. J Neurophysiol. 2003;90: 3921-3930. doi:10.1152/jn.00475.2003

25. Lockmann ALV, Laplagne DA, Tort ABL. Olfactory bulb drives respiration-coupled beta oscillations in the rat hippocampus. Eur $J$ Neurosci. 2018;48: 2663-2673. doi:https://doi.org/10.1111/ejn.13665

26. Gourévitch B, Kay LM, Martin C. Directional Coupling From the Olfactory Bulb to the Hippocampus During a Go/No-Go Odor Discrimination Task. J Neurophysiol. 2010;103: 2633-2641. doi:10.1152/jn.01075.2009 
bioRxiv preprint doi: https://doi.org/10.1101/2021.10.04.463041; this version posted October 21,2021 . The copyright holder for this preprint (which was not certified by peer review) is the author/funder. All rights reserved. No reuse allowed without permission.

27. Vanderwolf $\mathrm{CH}$, Zibrowski EM. Pyriform cortex $\beta$-waves: odor-specific sensitization following repeated olfactory stimulation. Brain Res. 2001;892: 301-308. doi:10.1016/S0006-8993(00)03263-7

28. $\mathrm{Xu} \mathrm{W}$, Wilson DA. Odor-evoked activity in the mouse lateral entorhinal cortex. Neuroscience. 2012;223: 12-20. doi:10.1016/j.neuroscience.2012.07.067

29. Igarashi KM, Lu L, Colgin LL, Moser M-B, Moser El. Coordination of entorhinalhippocampal ensemble activity during associative learning. Nature. 2014;510: 143-147. doi:10.1038/nature13162

30. Martin C, Gervais R, Messaoudi B, Ravel N. Learning-induced oscillatory activities correlated to odour recognition: a network activity. Eur J Neurosci. 2006;23: 1801-1810. doi:10.1111/j.1460-9568.2006.04711.x

31. Martin C, Beshel J, Kay LM. An Olfacto-Hippocampal Network Is Dynamically Involved in Odor-Discrimination Learning. J Neurophysiol. 2007;98: 2196-2205. doi:10.1152/jn.00524.2007

32. Ravel N, Chabaud P, Martin C, Gaveau V, Hugues E, Tallon-Baudry C, et al. Olfactory learning modifies the expression of odour-induced oscillatory responses in the gamma $(60-90 \mathrm{~Hz})$ and beta $(15-40 \mathrm{~Hz})$ bands in the rat olfactory bulb. Eur J Neurosci. 2003;17: 350-358. doi:https://doi.org/10.1046/j.1460-9568.2003.02445.x

33. Symanski CA, Bladon JH, Kullberg ET, Jadhav SP. Rhythmic coordination of hippocampal-prefrontal ensembles for odor-place associative memory and decision making. bioRxiv. 2021; 2020.06.08.140939. doi:10.1101/2020.06.08.140939

34. Gretenkord S, Kostka JK, Hartung H, Watznauer K, Fleck D, Minier-Toribio A, et al. Coordinated electrical activity in the olfactory bulb gates the oscillatory entrainment of entorhinal networks in neonatal mice. PLOS Biol. 2019;17: e2006994. doi:10.1371/journal.pbio.2006994

35. Kostka JK, Gretenkord S, Spehr M, Hanganu-Opatz IL. Bursting mitral cells time the oscillatory coupling between olfactory bulb and entorhinal networks in neonatal mice. $J$ Physiol. 2020;598: 5753-5769. doi:10.1113/JP280131

36. Siapas AG, Lubenov EV, Wilson MA. Prefrontal Phase Locking to Hippocampal Theta Oscillations. Neuron. 2005;46: 141-151. doi:10.1016/j.neuron.2005.02.028

37. Ramirez-Villegas JF, Besserve M, Murayama Y, Evrard HC, Oeltermann A, Logothetis NK. Coupling of hippocampal theta and ripples with pontogeniculooccipital waves. Nature. 2021;589: 96-102. doi:10.1038/s41586-020-2914-4

38. Shajarisales N, Janzing D, Shoelkopf B, Besserve M. Telling cause from effect in deterministic linear dynamical systems. ArXiv150301299 Cs. 2015 [cited 18 May 2021]. Available: http://arxiv.org/abs/1503.01299

39. Claudi F, Tyson AL, Petrucco L, Margrie TW, Portugues R, Branco T. Brainrender: a python-based software for visualizing anatomically registered data. bioRxiv. 2020; 2020.02.23.961748. doi:10.1101/2020.02.23.961748

40. Bitzenhofer SH, Ahlbeck J, Hanganu-Opatz IL. Methodological Approach for Optogenetic Manipulation of Neonatal Neuronal Networks. Front Cell Neurosci. 2017;11: 239. doi:10.3389/fncel.2017.00239 
bioRxiv preprint doi: https://doi.org/10.1101/2021.10.04.463041; this version posted October 21,2021 . The copyright holder for this preprint (which was not certified by peer review) is the author/funder. All rights reserved. No reuse allowed without permission.

851

852

853

854

855

856

857

858

859

860

861

862

863

864

865

866

867

868

869

870

871

872

873

874

875

876

877

878

879

880

881

882

883

884

885

886

887

888

889

890

891

41. Nolte G, Bai O, Wheaton L, Mari Z, Vorbach S, Hallett M. Identifying true brain interaction from EEG data using the imaginary part of coherency. Clin Neurophysiol. 2004;115: 2292-2307. doi:10.1016/j.clinph.2004.04.029

42. Thompson KJ, Khajehali E, Bradley SJ, Navarrete JS, Huang XP, Slocum S, et al. DREADD Agonist 21 Is an Effective Agonist for Muscarinic-Based DREADDs in Vitro and in Vivo. ACS Pharmacol Transl Sci. 2018;1: 61-72. doi:10.1021/acsptsci.8b00012

43. Roth BL. DREADDs for Neuroscientists. Neuron. 2016;89: 683-694. doi:10.1016/j.neuron.2016.01.040

44. Stachniak TJ, Ghosh A, Sternson SM. Chemogenetic synaptic silencing of neural circuits localizes a hypothalamus $\rightarrow$ midbrain pathway for feeding behavior. Neuron. 2014;82: 797-808. doi:10.1016/j.neuron.2014.04.008

45. Chini M, Hanganu-Opatz IL. Prefrontal Cortex Development in Health and Disease: Lessons from Rodents and Humans. Trends Neurosci. 2021;44: 227-240. doi:10.1016/j.tins.2020.10.017

46. Rio-Bermudez CD, Blumberg MS. Active Sleep Promotes Functional Connectivity in Developing Sensorimotor Networks. BioEssays. 2018;40: 1700234. doi:10.1002/bies.201700234

47. Welker WI. Analysis of Sniffing of the Albino Rat. Behaviour. 1964;22: 223-244. Available: https://www.jstor.org/stable/4533073

48. David F, Courtiol E, Buonviso N, Fourcaud-Trocmé N. Competing Mechanisms of Gamma and Beta Oscillations in the Olfactory Bulb Based on Multimodal Inhibition of Mitral Cells Over a Respiratory Cycle. eNeuro. 2015;2. doi:10.1523/ENEURO.001815.2015

49. Fourcaud-Trocmé N, Courtiol E, Buonviso N. Two distinct olfactory bulb sublaminar networks involved in gamma and beta oscillation generation: a CSD study in the anesthetized rat. Front Neural Circuits. 2014;8. doi:10.3389/fncir.2014.00088

50. Osinski BL, Kay LM. Granule cell excitability regulates gamma and beta oscillations in a model of the olfactory bulb dendrodendritic microcircuit. J Neurophysiol. 2016;116: 522539. doi:10.1152/jn.00988.2015

51. Osinski BL, Kim A, Xiao W, Mehta NM, Kay LM. Pharmacological manipulation of the olfactory bulb modulates beta oscillations: testing model predictions. J Neurophysiol. 2018;120: 1090-1106. doi:10.1152/jn.00090.2018

52. Dietz SB, Markopoulos F, Murthy VN. Postnatal Development of Dendrodendritic Inhibition in the Mammalian Olfactory Bulb. Front Cell Neurosci. 2011;5. doi:10.3389/fncel.2011.00010

53. Fletcher ML, Smith AM, Best AR, Wilson DA. High-Frequency Oscillations Are Not Necessary for Simple Olfactory Discriminations in Young Rats. J Neurosci. 2005;25: 792798. doi:10.1523/JNEUROSCI.4673-04.2005

54. Yu Y, Burton SD, Tripathy SJ, Urban NN. Postnatal development attunes olfactory bulb mitral cells to high-frequency signaling. J Neurophysiol. 2015;114: 2830-2842. doi:10.1152/jn.00315.2015 
bioRxiv preprint doi: https://doi.org/10.1101/2021.10.04.463041; this version posted October 21, 2021. The copyright holder for this preprint (which was not certified by peer review) is the author/funder. All rights reserved. No reuse allowed without permission.

55. Bitzenhofer SH, Pöpplau JA, Hanganu-Opatz I. Gamma activity accelerates during prefrontal development. Vinck M, Colgin LL, Perrenoud Q, Moore Cl, editors. eLife. 2020;9: e56795. doi:10.7554/eLife.56795

56. Chen G, Rasch MJ, Wang R, Zhang X. Experience-dependent emergence of beta and gamma band oscillations in the primary visual cortex during the critical period. Sci Rep. 2015;5: 17847. doi:10.1038/srep17847

57. Hoy JL, Niell CM. Layer-Specific Refinement of Visual Cortex Function after Eye Opening in the Awake Mouse. J Neurosci. 2015;35: 3370-3383.

58. Schwerdtfeger WK, Buhl EH, Germroth P. Disynaptic olfactory input to the hippocampus mediated by stellate cells in the entorhinal cortex. J Comp Neurol. 1990;292: 163-177. doi:https://doi.org/10.1002/cne.902920202

59. Wouterlood FG, Nederlof J. Terminations of olfactory afferents on layer II and III neurons in the entorhinal area: degeneration-Golgi-electron microscopic study in the rat. Neurosci Lett. 1983;36: 105-110. doi:10.1016/0304-3940(83)90250-1

60. Li Y, Xu J, Liu Y, Zhu J, Liu N, Zeng W, et al. A distinct entorhinal cortex to hippocampal CA1 direct circuit for olfactory associative learning. Nat Neurosci. 2017;20: 559-570. doi: $10.1038 / \mathrm{nn} .4517$

61. Walz A, Omura M, Mombaerts P. Development and topography of the lateral olfactory tract in the mouse: Imaging by genetically encoded and injected fluorescent markers. $J$ Neurobiol. 2006;66: 835-846. doi:https://doi.org/10.1002/neu.20266

62. Basu J, Zaremba JD, Cheung SK, Hitti FL, Zemelman BV, Losonczy A, et al. Gating of hippocampal activity, plasticity, and memory by entorhinal cortex long-range inhibition. Science. 2016;351. doi:10.1126/science.aaa5694

63. Spitzer B, Haegens S. Beyond the Status Quo: A Role for Beta Oscillations in Endogenous Content (Re)Activation. eNeuro. 2017;4: ENEURO.0170-17.2017. doi:10.1523/ENEURO.0170-17.2017

64. Rangel LM, Rueckemann JW, Riviere PD, Keefe KR, Porter BS, Heimbuch IS, et al. Rhythmic coordination of hippocampal neurons during associative memory processing. Bartos M, editor. eLife. 2016;5: e09849. doi:10.7554/eLife.09849

65. Iwasaki S, Sasaki T, Ikegaya Y. Hippocampal beta oscillations predict mouse objectlocation associative memory performance. Hippocampus. 2021;31: 503-511. doi:10.1002/hipo.23311

66. Bibbig A, Traub RD, Whittington MA. Long-range synchronization of gamma and beta oscillations and the plasticity of excitatory and inhibitory synapses: a network model. $\mathrm{J}$ Neurophysiol. 2002;88: 1634-1654. doi:10.1152/jn.2002.88.4.1634

67. Kopell N, Ermentrout GB, Whittington MA, Traub RD. Gamma rhythms and beta rhythms have different synchronization properties. Proc Natl Acad Sci. 2000;97: 1867-1872. doi:10.1073/pnas.97.4.1867

68. Kopell N, Whittington MA, Kramer MA. Neuronal assembly dynamics in the beta1 frequency range permits short-term memory. Proc Natl Acad Sci. 2011;108: 3779-3784. doi:10.1073/pnas.1019676108 
69. Cichon NB, Denker M, Grün S, Hanganu-Opatz IL. Unsupervised classification of neocortical activity patterns in neonatal and pre-juvenile rodents. Front Neural Circuits. 2014;8. doi:10.3389/fncir.2014.00050

70. Rossant C, Kadir SN, Goodman DFM, Schulman J, Hunter MLD, Saleem AB, et al. Spike sorting for large, dense electrode arrays. Nat Neurosci. 2016;19: 634-641. doi:10.1038/nn.4268

71. Tort ABL, Komorowski R, Eichenbaum H, Kopell N. Measuring Phase-Amplitude Coupling Between Neuronal Oscillations of Different Frequencies. J Neurophysiol. 2010;104: 1195-1210. doi:10.1152/jn.00106.2010 


\section{Supplementary Figures}

A

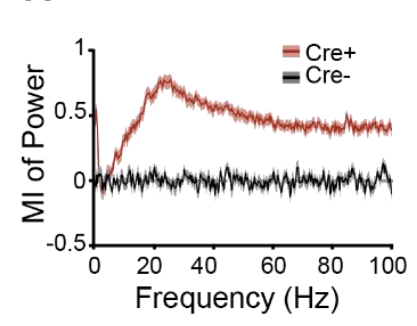

B
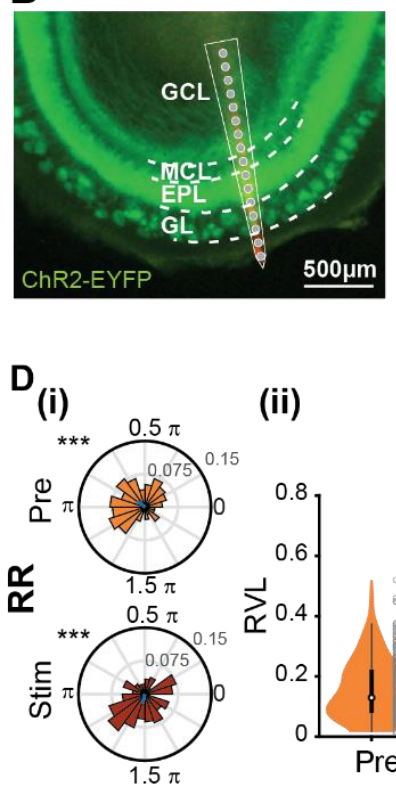

(ii)
C
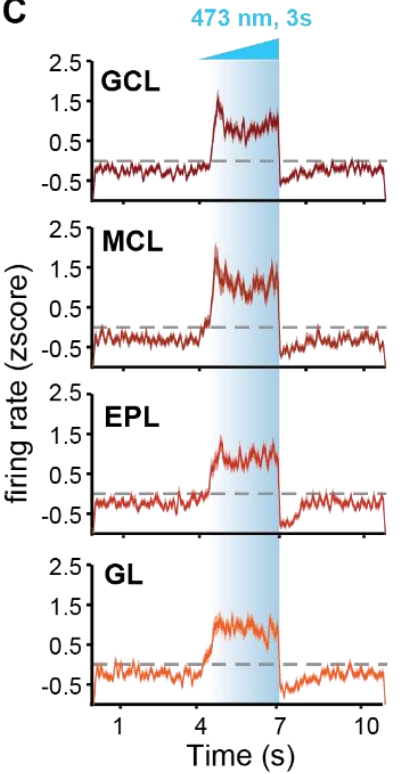

(iii)

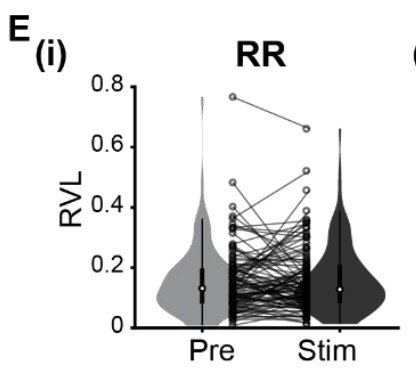

(ii)

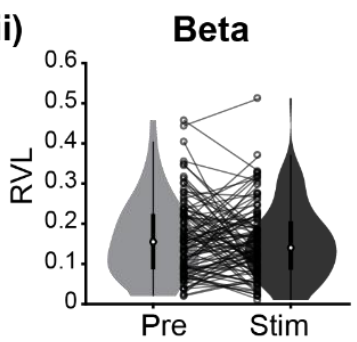

S1 Fig. (related to Fig 2). OB activity in response to ramp stimulation

(A) Plot of MI for LFP power in OB during ramp stimulation for $\mathrm{cre}^{+}$(red) and cre- (black) mice. (B) Digital photomontages displaying the Dil-labeled electrode track in a coronal slice including the ChR2-EYFP-expressing OB. Gray dots correspond to the individual recording sites. Dashed white lines mark borders between the different $O B$ layers. (C) Z-scored firing rate of units recorded in the different $\mathrm{OB}$ layers in response to light stimulation. (D) (i) Polar plots displaying phase locking of $O B$ units to $R R$ before (Pre, orange) and during ramp stimulation (Stim, red) for $\mathrm{cre}^{+}$mice. The mean resulting vectors are displayed as a blue line. ${ }^{* * *} p<$ 0.001 , Rayleigh test for non-uniformity). (ii) Violin plots displaying the RVL of OB units before (Pre, orange) and during ramp stimulation (Stim, red). Gray dots and lines correspond to individual units. ( ${ }^{* * *} p<0.001$, linear-mixed model). (iii) Bar plots displaying the percentage of significantly locked units before (Pre, yellow) and during (Stim, red) stimulation. ( ${ }^{*} p<0.05$, Fisher's exact test). (E) (i) Violin plots displaying the RVL of $O B$ units locked to RR before stimulation (Pre, gray) and during ramp stimulation (Stim, black) for cremice. Black dots and lines correspond to individual units. ( ${ }^{*} p<0.05$, linear mixed-effect model). (ii) Same as (i) for beta oscillations. 
A (i)

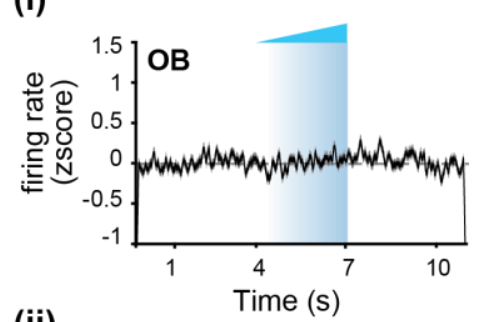

(ii)

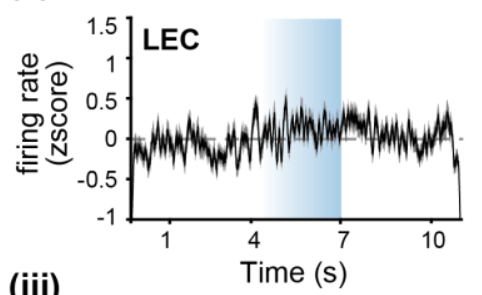

(iii)

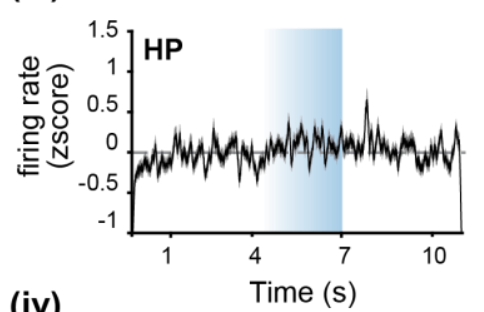

(iv)

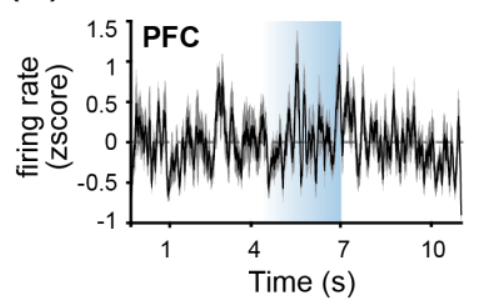

B (i)

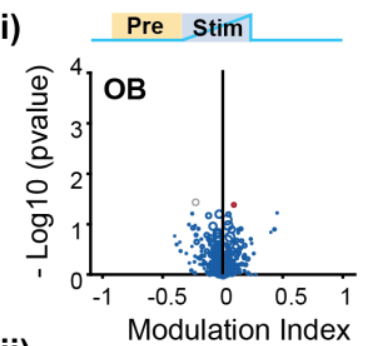

(ii)
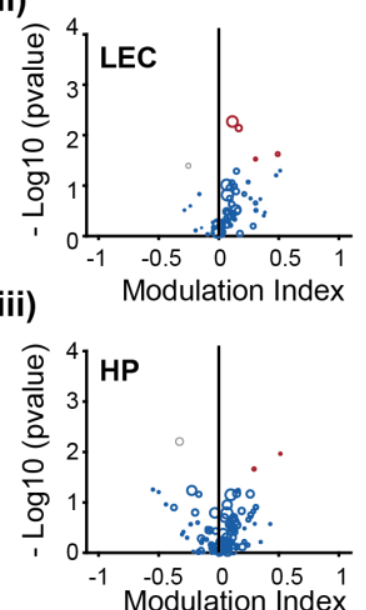

(iv)

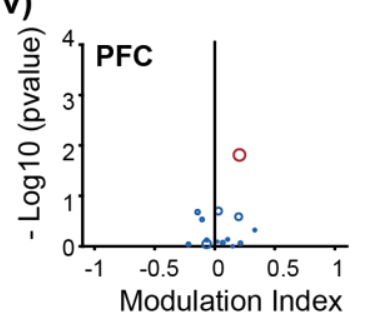

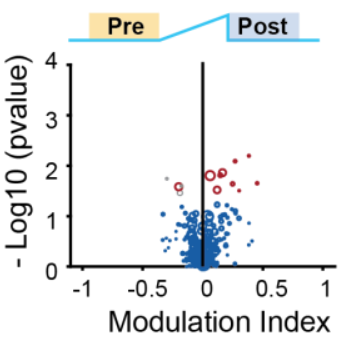
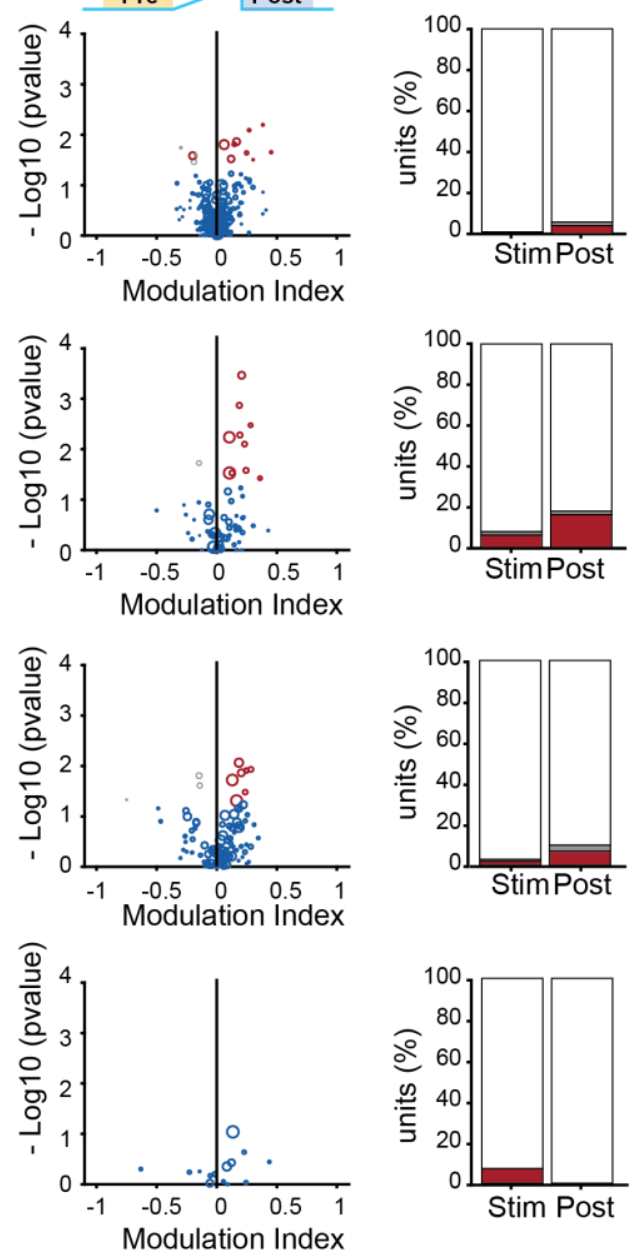

S2 Fig. (related to Figs 2 and 3). Effects of optogenetic manipulation of M/TCs from cre- mice on single-unit activity.

(A) (i) Z-scored firing rate of units recorded in the $\mathrm{OB}$ of cre- (black) mice in response to light stimulation. (ii) Same as (i) for units recorded in LEC. (iii) Same as (i) for units recorded in HP. (iv) Same as (i) for units recorded in PFC. (B) (i) Left, volcano plot displaying the MI of SUA firing rates recorded in the OB of cre- mice before (Pre) vs. during (Stim) ramp stimulation (significant activated units are shown in red and significant inhibited units in gray, $p<0.01$, Wilcoxon signed-rank test). Middle, same as the left image but for SUA firing rates before (Pre) vs. after (Post) ramp stimulation. Right, bar plots depicting the percentage of activated (red) and inhibited (gray) units during (Stim) and after (Post) ramp stimulation. (ii) Same as (i) for units recorded in LEC. (iii) Same as (i) for units recorded in HP. (iv) Same as (i) for units recorded in PFC. 
A

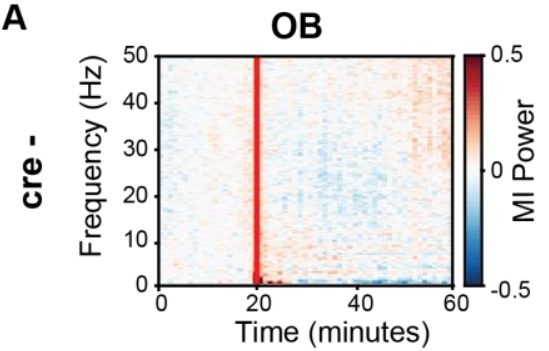

в

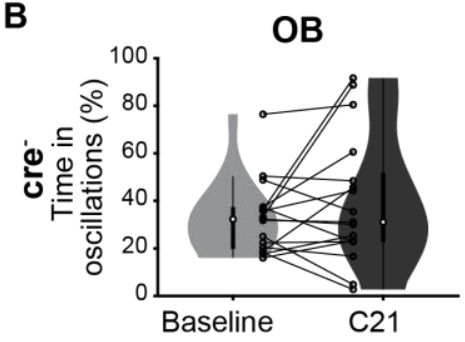

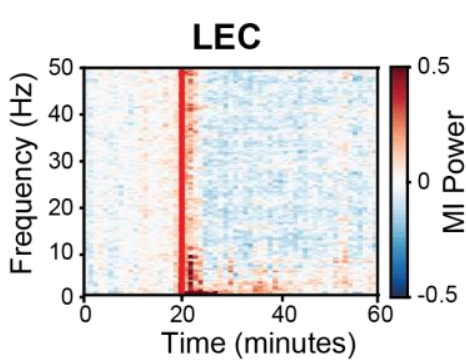

LEC

C
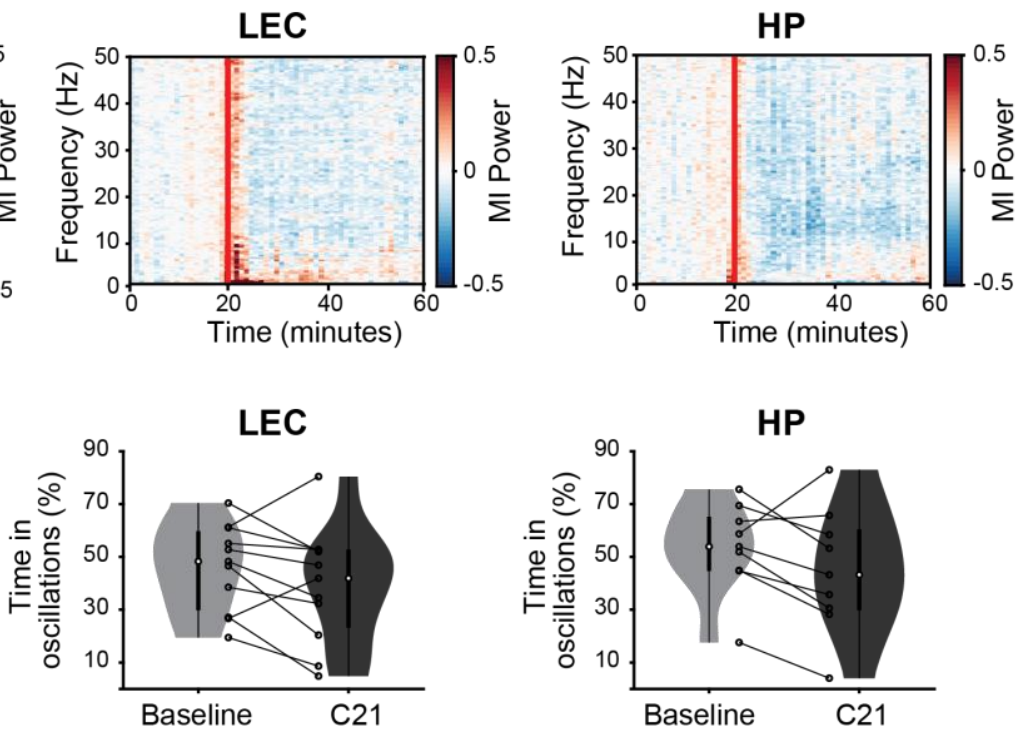

OB

LEC

HP
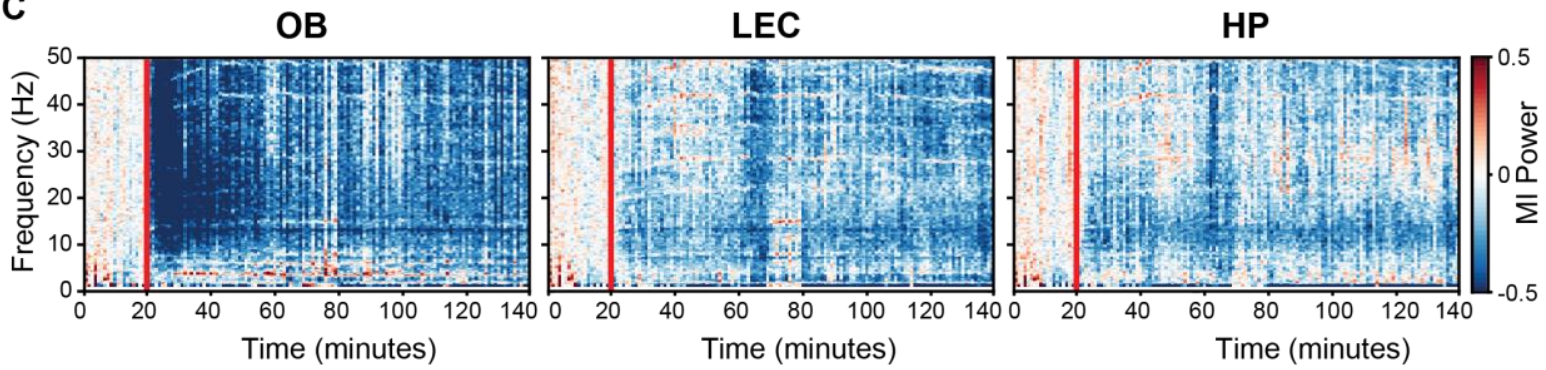

S3 Fig. (related to Fig 5). Effects of Compound 21 injection in cre- mice and long-lasting effects of $M / T C$ silencing by inhibitory DREADDs.

(A) Color-coded MI of LFP power before and after C21 injection in OB (left), LEC (middle), and HP (right) of cre- mice. The red line corresponds to the C21 injection. (B) Violin plots displaying the percentage of time spend in discontinuous oscillatory events before (Baseline, gray) and after C21 injection (C21, black) for cre- mice. Black dots and lines correspond to individual animals. ${ }^{*} p<0.05$, Wilcoxon signed-rank test). (C) Color-coded MI of LFP power before and after (120 min) C21 injection in OB (left), LEC (middle) and HP (right) of cre+ mice $(n=3)$. The red line corresponds to the C21 injection. 
A (i)

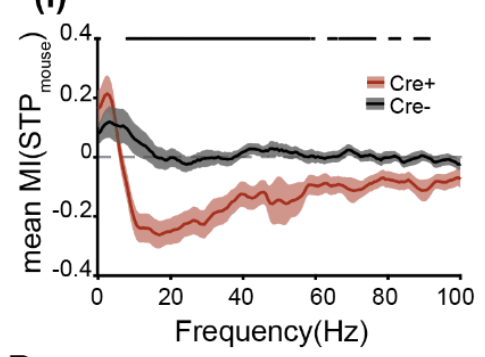

B

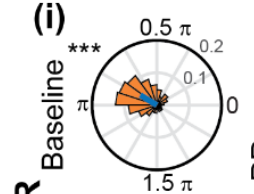

$\frac{\kappa}{\sim}$
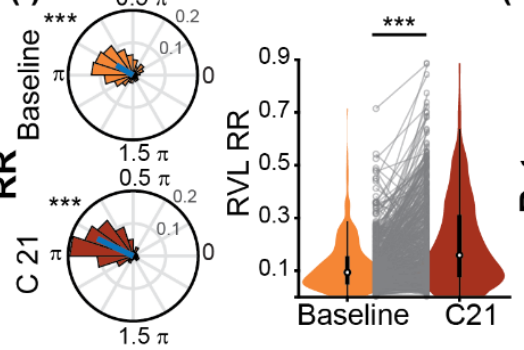

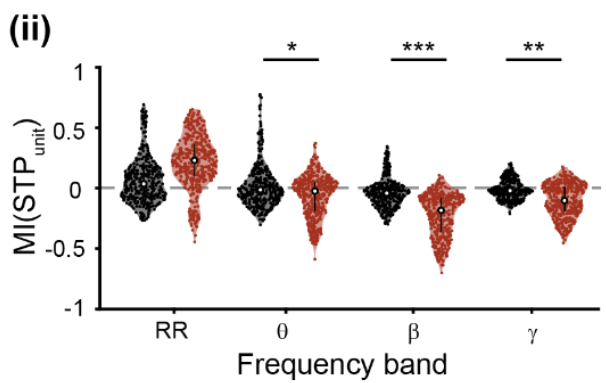

(ii)
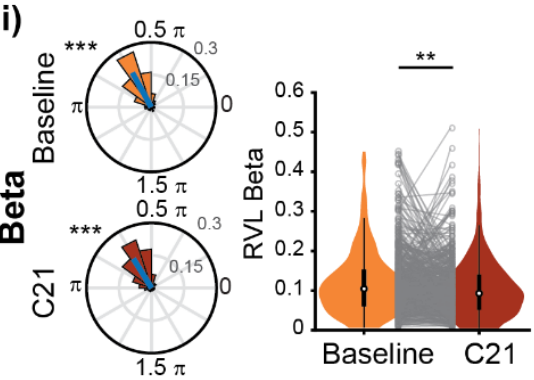

S4 Fig. (related to Fig 5). STP and phase locking of OB firing to LFP oscillations in OB after silencing the MT/C output by inhibitory DREADDs

(A) (i) Plot of Ml of STP for cre $^{+}$(red) and cre- (black) mice before and after C21 injection in OB. (black line: $p<0.05$, Wilcoxon signed-rank test). (ii) Violin plots displaying mean MI for STP for each OB unit for different frequency bands for $\mathrm{cre}^{+}$(red) and cre- (black) mice. Red and black dots correspond to individual units. ( $p<0.05,{ }^{* *} p<0.01$, ${ }^{* * *} p<0.001$, linear mixed-effect model). (B) (i) Right, phase locking of $\mathrm{OB}$ units to RR oscillations in $\mathrm{OB}$. Left, polar plots displaying phase locking of $\mathrm{OB}$ units before (Baseline, orange) and after $\mathrm{C} 21$ injection (C21, red). The mean resulting vectors are represented as a blue line. ( ${ }^{* \star *} p<0.001$, Rayleigh test for non-uniformity). Right, violin plots displaying the RVL of OB units before (Baseline, orange) and after $\mathrm{C} 21$ injection (C21, red). Gray dots and lines correspond to individual units. (ii) Same as (i) for beta oscillations in the $O B\left({ }^{* *} p<0.01\right.$, ${ }^{* * *} p<0.001$, linear mixedeffect model) 
A

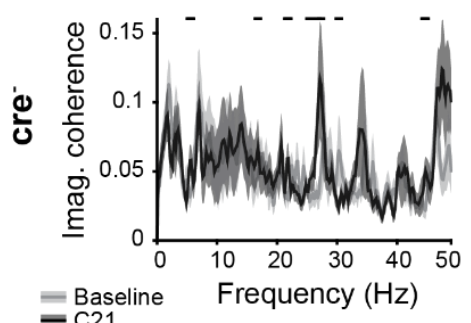

LEC-HP

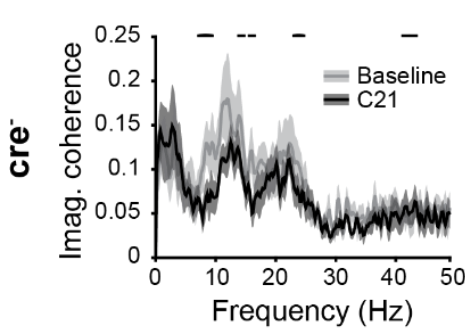

OB-HP

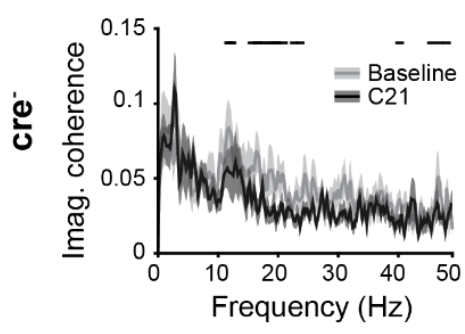

B
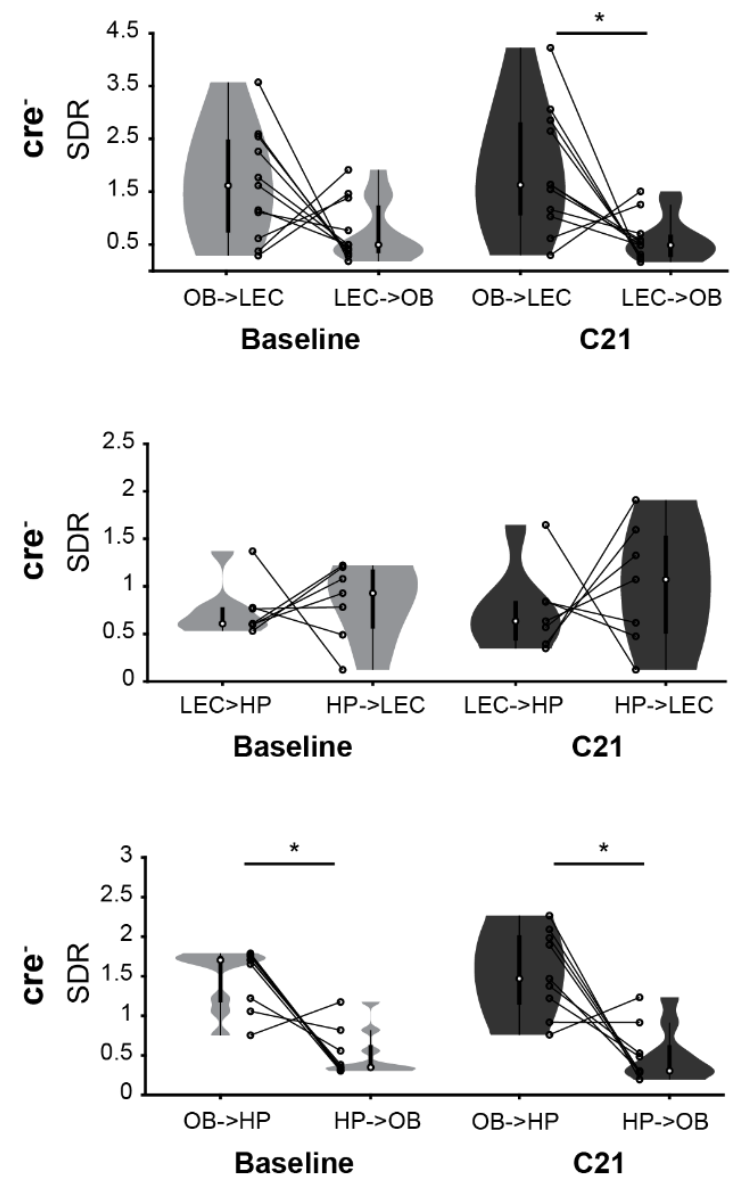

S5 Fig. (related to Fig 6). Effects of Compound 21 injection on oscillatory coupling in cre- mice

(A) Imaginary coherence calculated for OB - LEC (top), LEC - HP (middle) and OB - HP (bottom) in cremice, before (Baseline, gray) and after $\mathrm{C} 21$ injection (C21, black). (black line: $p<0.05$, Wilcoxon ranksum test). (B) SDR for OB and LEC (top): SDR values for OB -> LEC and LEC -> OB before (Baseline, gray) and after $\mathrm{C} 21$ injection (C21, black). Black dots and lines correspond to individual animals. Middle, same for LEC and HP. Bottom, same for OB and HP. ( ${ }^{*} p<0.05$, Wilcoxon signed-rank test). 


\section{Supplementary Data Tables}

\section{Phase p}

(circ. mean \pm circ. SEM, \% of sig. locked (Rayleigh test for nonunits) uniformity)

\begin{tabular}{lll}
\hline OB & $0.763 \pi \pm 0.006 \pi$ & $\mathrm{p}=7.6^{*} 10^{-297}$ \\
& $640 / 1124$ units $(56.94 \%)$ & \\
\hline LEC & $1.556 \pi \pm 0.038 \pi$ & $\mathrm{p}=4.19^{*} 10^{-11}$ \\
& $42 / 384$ units $(10.94 \%)$ & \\
\hline HP & $0.315 \pi \pm 0.047 \pi$ & $\mathrm{p}=3.68^{*} 10^{-5}$ \\
& $51 / 384$ units $(13.28 \%)$ & $\mathrm{p}=0.605$ \\
\hline PFC & $1.715 \pi \pm 0.082 \pi$ & \\
& $26 / 318$ units $(8.18 \%)$ &
\end{tabular}

S1 Table (related to Fig 1D). Phase locking of SUA to OB beta phase.

1. Brain area

(circ. mean \pm circ. SEM)
2. Brain area (circ. mean \pm circ. SEM)

\section{p}

(Non-parametric multisample test for equal medians)

\begin{tabular}{lllll}
\hline OB & $0.763 \pi \pm 0.006 \pi$ & LEC & $1.556 \pi \pm 0.038 \pi$ & $\mathrm{p}=4.58^{*} 10^{-4}$ \\
& & HP & $0.315 \pi \pm 0.047 \pi$ & $\mathrm{p}=2.33^{*} 10^{-5}$ \\
& & PFC & $1.715 \pi \pm 0.082 \pi$ & $\mathrm{p}=0.689$ \\
\hline LEC & $1.556 \pi \pm 0.038 \pi$ & HP & $0.315 \pi \pm 0.047 \pi$ & $\mathrm{p}=9.44^{*} 10^{-9}$ \\
& & PFC & $1.715 \pi \pm 0.082 \pi$ & $\mathrm{p}=0.618$ \\
\hline HP & $0.315 \pi \pm 0.047 \pi$ & PFC & $1.715 \pi \pm 0.082 \pi$ & $\mathrm{p}=0.045$
\end{tabular}

S2 Table (related to Fig 1D). Phase angles of significant locked SUA to OB beta phase. 
Area $1 \rightarrow$ Area 2

(median, interquartile range)
Area $2 \rightarrow$ Area 1

(median, interquartile range) p

(Wilcoxon signedrank test)

\begin{tabular}{llll}
\hline $\begin{array}{l}\text { Area 1: OB } \\
\text { Area 2: LEC }\end{array}$ & $2.051[1.666-2.580]$ & $0.407[0.262-0.522]$ & $\mathrm{p}=5.68^{*} 10^{-8}(\mathrm{n}=39)$ \\
\hline $\begin{array}{l}\text { Area 1: OB } \\
\text { Area 2: HP }\end{array}$ & $1.389[0.920-1.868]$ & $0.518[0.334-0.657]$ & $\mathrm{p}=2.52^{*} 10^{-5}(\mathrm{n}=38)$ \\
\hline $\begin{array}{l}\text { Area 1: OB } \\
\text { Area 2: PFC }\end{array}$ & $2.809[1.925-3.760]$ & $0.309[0.226-0.399]$ & $\mathrm{p}=8.39^{*} 10^{-8}(\mathrm{n}=38)$ \\
\hline $\begin{array}{l}\text { Area 1: LEC } \\
\text { Area 2: HP }\end{array}$ & $0.650[0.509-1.019]$ & $1.022[0.831-1.372]$ & $\mathrm{p}=0.056(\mathrm{n}=28)$ \\
\hline $\begin{array}{l}\text { Area 1: LEC } \\
\text { Area 2: PFC }\end{array}$ & $1.188[0.895-1.375]$ & $0.762[0.635-1.045]$ & $\mathrm{p}=0.024(\mathrm{n}=30)$ \\
\hline $\begin{array}{l}\text { Area 1: HP } \\
\text { Area 2: PFC }\end{array}$ & $1.717[1.317-2.034]$ & $0.481[0.344-0.647]$ & $\mathrm{p}=0.0006(\mathrm{n}=24)$ \\
& & &
\end{tabular}

S3 Table (related to Fig 1F). SDR for pairs of brain areas

\begin{tabular}{llll}
$\begin{array}{l}\text { Cre- } \\
\left(\begin{array}{l}\text { nunits }_{100} \text { from } \\
13 \text { mice }\end{array}\right.\end{array}$ & $\begin{array}{l}\text { Pre } \\
\text { (median, interquartile } \\
\text { range) }\end{array}$ & $\begin{array}{l}\text { Stim } \\
\text { (median, interquartile } \\
\text { range) }\end{array}$ & $\begin{array}{l}\mathbf{p} \\
\text { (linear fixed- } \\
\text { effect model) }\end{array}$ \\
\hline RR [2-4 Hz] & $0.131[0.083-0.196]$ & $0.128[0.085-0.208]$ & $p=0.763$ \\
\hline Beta [12-30 Hz] & $0.155[0.088-0.222]$ & $0.140[0.087-0.205]$ & $p=0.486$
\end{tabular}

S4 Table (related to S1E Fig). Mean resulting vector length for phase locking of OB firing to RR and beta oscillations for cre- mice. 


\begin{tabular}{|c|c|c|c|c|}
\hline & & $\begin{array}{l}\mathrm{Cre}^{+} \\
\text {(median, interquartile } \\
\text { range, Wilcoxon signed- } \\
\text { rank test) }\end{array}$ & $\begin{array}{l}\mathrm{Cre}^{-} \\
\text {(median, interquartile } \\
\text { range, Wilcoxon signed- } \\
\text { rank test) }\end{array}$ & $\begin{array}{l}\mathbf{p} \\
\text { (Wilcoxon } \\
\text { rank-sum test) }\end{array}$ \\
\hline \multirow[t]{4}{*}{ OB } & $R R[2-4 \mathrm{~Hz}]$ & $\begin{array}{l}-0.055[-0.202-0.039] \\
p=0.073(n=32)\end{array}$ & $\begin{array}{l}-0.011[-0.061-0.029] \\
p=0.463(n=14)\end{array}$ & $p=0.397$ \\
\hline & Theta [4-12 Hz] & $\begin{array}{l}0.199[0.056-0.322] \\
p=4.96^{*} 10^{-5}(n=32)\end{array}$ & $\begin{array}{l}-0.003[-0.023-0.047] \\
p=0.952(n=14)\end{array}$ & $p=0.001$ \\
\hline & Beta [12-30 Hz] & $\begin{array}{l}0.586[0.336-0.679] \\
p=7.95^{\star} 10^{-7}(n=32)\end{array}$ & $\begin{array}{l}0.004[-0.036-0.018] \\
p=1(n=14)\end{array}$ & $p=9.52^{*} 10^{-8}$ \\
\hline & Gamma [30-100 Hz] & $\begin{array}{l}0.555[0.292-0.661] \\
p=7.95^{\star} 10^{-7}(n=32)\end{array}$ & $\begin{array}{l}-0.005[-0.037-0.022] \\
p=0.670(n=14)\end{array}$ & $p=9.52^{*} 10^{-8}$ \\
\hline \multirow[t]{4}{*}{ LEC } & RR [2-4 Hz] & $\begin{array}{l}0.111[-0.032-0.225] \\
p=0.019(n=22)\end{array}$ & $\begin{array}{l}0.096[0.008-0.184] \\
p=0.064(n=10)\end{array}$ & $p=0.984$ \\
\hline & Theta [4-12 Hz] & $\begin{array}{l}0.097[-0.019-0.201] \\
p=0.004(n=22)\end{array}$ & $\begin{array}{l}0.065[0.032-0.117] \\
0.002(n=10)\end{array}$ & $\mathrm{p}=0.760$ \\
\hline & Beta [12-30 Hz] & $\begin{array}{l}0.058[0.021-0.118] \\
p=0.0005(n=22)\end{array}$ & $\begin{array}{l}0.030[-0.013-0.054] \\
p=0.275(n=10)\end{array}$ & $p=0.044$ \\
\hline & Gamma [30-100 Hz] & $\begin{array}{l}0.044[0.013-0.101] \\
\mathrm{p}=0.0008(\mathrm{n}=22)\end{array}$ & $\begin{array}{l}0.020[-0.014-0.033] \\
p=0.375(n=10)\end{array}$ & $p=0.070$ \\
\hline \multirow[t]{4}{*}{ HP } & RR [2-4 Hz] & $\begin{array}{l}0.002[-0.113-0.222] \\
p=0.661(n=22)\end{array}$ & $\begin{array}{l}0.073[-0.052-0.110] \\
p=0.206(n=11)\end{array}$ & $p=0.580$ \\
\hline & Theta [4-12 Hz] & $\begin{array}{l}0.043[0.014-0.186] \\
p=0.002(n=22)\end{array}$ & $\begin{array}{l}0.003[-0.022-0.098] \\
p=0.413(n=11)\end{array}$ & $p=0.152$ \\
\hline & Beta [12-30 Hz] & $\begin{array}{l}0.061[0.038-0.105] \\
\mathrm{p}=0.000983(\mathrm{n}=22)\end{array}$ & $\begin{array}{l}0.007[-0.021-0.045] \\
p=0.465(n=11)\end{array}$ & $p=0.049$ \\
\hline & Gamma [30-100 Hz] & $\begin{array}{l}0.067[0.016-0.114] \\
\mathrm{p}=0.001(\mathrm{n}=22)\end{array}$ & $\begin{array}{l}0.042[-0.028-0.057] \\
p=0.206(n=11)\end{array}$ & $p=0.089$ \\
\hline \multirow[t]{4}{*}{ PFC } & RR [2-4 Hz] & $\begin{array}{l}0.020[-0.055-0.252] \\
p=0.211(n=22)\end{array}$ & $\begin{array}{l}0.017[-0.033-0.191] \\
p=0.461(n=8)\end{array}$ & $p=0.981$ \\
\hline & Theta [4-12 Hz] & $\begin{array}{l}0.083[0.014-0.172] \\
p=0.006(n=22)\end{array}$ & $\begin{array}{l}0.032[-0.022-0.068] \\
p=0.25(n=8)\end{array}$ & $p=0.336$ \\
\hline & Beta [12-30 Hz] & $\begin{array}{l}0.080[0.025-0.116] \\
p=0.0002(n=22)\end{array}$ & $\begin{array}{l}0.004[-0.021-0.049] \\
p=0.641 \quad(n=8)\end{array}$ & $p=0.023$ \\
\hline & Gamma [30-100 Hz] & $\begin{array}{l}0.061[-0.007-0.084] \\
p=0.002(n=22)\end{array}$ & $\begin{array}{l}0.010[-0.011-0.064] \\
p=0.383(n=8)\end{array}$ & $p=0.336$ \\
\hline
\end{tabular}

S5 Table (related to Figs 2D and 4B). MI of power for cre $^{+}$and cre` animals during light stimulation. 


\begin{tabular}{|c|c|c|c|c|}
\hline & & $\begin{array}{l}\mathrm{Cre}^{+} \\
\text {(median, interquartile } \\
\text { range, } \quad \text { Wilcoxon } \\
\text { signed-rank test) }\end{array}$ & $\begin{array}{l}\mathrm{Cre}^{-} \\
\text {(median, interquartile } \\
\text { range, Wilcoxon signed- } \\
\text { rank test) }\end{array}$ & $\begin{array}{l}\mathbf{p} \\
\text { (Wilcoxon } \\
\text { rank-sum } \\
\text { test) }\end{array}$ \\
\hline \multirow[t]{4}{*}{ OB-LEC } & RR [2-4 Hz] & $\begin{array}{l}0.010[-0.130-0.304] \\
p=0.426(n=22)\end{array}$ & $\begin{array}{l}0.019[-0.146-0.194] \\
p=0.695(n=10)\end{array}$ & $p=0.984$ \\
\hline & Theta [4-12 Hz] & $\begin{array}{l}-0.030[-0.136-0.078] \\
p=0.426(n=22)\end{array}$ & $\begin{array}{l}0.029[-0.035-0.073] \\
p=0.432(n=10)\end{array}$ & $p=0.281$ \\
\hline & Beta [12-30 Hz] & $\begin{array}{l}0.109[-0.013-0.208] \\
p=0.013(n=22)\end{array}$ & $\begin{array}{l}0.010[-0.028-0.041] \\
p=0.922(n=10)\end{array}$ & $p=0.033$ \\
\hline & Gamma [30-100 Hz] & $\begin{array}{l}-0.002[-0.078-0.036] \\
p=0.249(n=22)\end{array}$ & $\begin{array}{l}0.010[-0.025-0.033] \\
p=0.846(n=10)\end{array}$ & $p=0.529$ \\
\hline \multirow[t]{4}{*}{ OB-HP } & RR [2-4 Hz] & $\begin{array}{l}0.062[-0.093-0.230] \\
\mathrm{p}=0.223(\mathrm{n}=22)\end{array}$ & $\begin{array}{l}0.103[-0.191-0.280] \\
p=0.577(n=11)\end{array}$ & $p=0.924$ \\
\hline & Theta [4-12 Hz] & $\begin{array}{l}0.036[-0.060-0.143] \\
p=0.223(n=22)\end{array}$ & $\begin{array}{l}-0.041[-0.235-0.061] \\
0.365(n=11)\end{array}$ & $p=0.122$ \\
\hline & Beta [12-30 Hz] & $\begin{array}{l}0.081[-0.018-0.139] \\
\mathrm{p}=0.042(\mathrm{n}=22)\end{array}$ & $\begin{array}{l}-0.048[-0.149-0.067] \\
p=0.519(n=11)\end{array}$ & $p=0.070$ \\
\hline & Gamma [30-100 Hz] & $\begin{array}{l}-0.028[-0.067-0.016] \\
p=0.082(n=22)\end{array}$ & $\begin{array}{l}0.022[-0.036-0.054] \\
p=0.700(n=11)\end{array}$ & $p=0.113$ \\
\hline \multirow[t]{4}{*}{ OB-PFC } & RR [2-4 Hz] & $\begin{array}{l}-0.091[-0.253-0.173] \\
p=0.322(n=22)\end{array}$ & $\begin{array}{l}-0.187[-0.244-0.013] \\
p=0.383(n=8)\end{array}$ & $p=0.439$ \\
\hline & Theta [4-12 Hz] & $\begin{array}{l}-0.006[-0.096-0.107] \\
p=0.910(n=22)\end{array}$ & $\begin{array}{l}-0.017[-0.200-0.099] \\
p=0.742(n=8)\end{array}$ & $p=0.725$ \\
\hline & Beta [12-30 Hz] & $\begin{array}{l}0.213[0.040-0.256] \\
p=0.0004(n=22)\end{array}$ & $\begin{array}{l}-0.022[-0.112-0.068] \\
p=0.641(n=8)\end{array}$ & $p=0.011$ \\
\hline & Gamma [30-100 Hz] & $\begin{array}{l}0.038[0.013-0.085] \\
p=0.009(n=22)\end{array}$ & $\begin{array}{l}-0.020[-0.059-0.036] \\
p=0.641(n=8)\end{array}$ & $p=0.071$ \\
\hline
\end{tabular}

S6 Table (related to Fig 4C). MI of coherence for cre ${ }^{+}$and cre- animals after C21 injection. 


\begin{tabular}{|c|c|c|c|c|}
\hline & & $\begin{array}{l}\mathrm{Cre}^{+} \\
\text {(median, interquartile } \\
\text { range, Wilcoxon signed- } \\
\text { rank test) }\end{array}$ & $\begin{array}{l}\text { Cre- }^{-} \\
\text {(median, interquartile } \\
\text { range, Wilcoxon signed- } \\
\text { rank test) }\end{array}$ & $\begin{array}{l}\mathbf{p} \\
\text { (Wilcoxon } \\
\text { rank-sum test) }\end{array}$ \\
\hline \multirow[t]{4}{*}{ OB } & RR [2-4 Hz] & $\begin{array}{l}-0.032[-0.160-0.157] \\
p=0.687(n=17)\end{array}$ & $\begin{array}{l}-0.025[-0.084-0.116] \\
p=0.879(n=18)\end{array}$ & $p=0.609$ \\
\hline & Theta [4-12 Hz] & $\begin{array}{l}-0.082[-0.286--0.014] \\
p=0.013(n=17)\end{array}$ & $\begin{array}{l}-0.010[-0.094-0.156] \\
p=0.472(n=18)\end{array}$ & $p=0.031$ \\
\hline & Beta [12-30 Hz] & $\begin{array}{l}-0.246[-0.426--0.117] \\
p=0.0003(n=17)\end{array}$ & $\begin{array}{l}-0.025[-0.137-0.052] \\
p=0.349(n=18)\end{array}$ & $p=0.0003$ \\
\hline & Gamma [30-100 Hz] & $\begin{array}{l}-0.162[-0.225-0.006] \\
p=0.006(n=17)\end{array}$ & $\begin{array}{l}-0.009[-0.083-0.040] \\
p=0.647(n=18)\end{array}$ & $p=0.028$ \\
\hline \multirow[t]{4}{*}{ LEC } & RR [2-4 Hz] & $\begin{array}{l}-0.337[-0.492--0.109] \\
p=0.002(n=13)\end{array}$ & $\begin{array}{l}0.246[-0.096-0.488] \\
p=0.063(n=12)\end{array}$ & $\mathrm{p}=0.000997$ \\
\hline & Theta [4-12 Hz] & $\begin{array}{l}-0.293[-0.435--0.10] \\
p=0.001(n=13)\end{array}$ & $\begin{array}{l}0.080[-0.126-0.330] \\
0.259(n=12)\end{array}$ & $p=0.0008$ \\
\hline & Beta [12-30 Hz] & $\begin{array}{l}-0.195[-0.424--0.115] \\
p=0.0002(n=13)\end{array}$ & $\begin{array}{l}-0.043[-0.106-0.025] \\
p=0.226(n=12)\end{array}$ & $p=0.0005$ \\
\hline & Gamma [30-100 Hz] & $\begin{array}{l}-0.106[-0.151--0.044] \\
p=0.0005(n=13)\end{array}$ & $\begin{array}{l}-0.015[-0.050-0.323] \\
p=0.663(n=12)\end{array}$ & $p=0.004$ \\
\hline \multirow[t]{4}{*}{ HP } & RR [2- $4 \mathrm{~Hz}]$ & $\begin{array}{l}-0.131[-0.285--0.047] \\
p=0.049(n=10)\end{array}$ & $\begin{array}{l}-0.074[-0.160-0.036] \\
p=0.426(n=9)\end{array}$ & $p=0.243$ \\
\hline & Theta [4-12 Hz] & $\begin{array}{l}-0.202[-0.332--0.144] \\
p=0.006(n=10)\end{array}$ & $\begin{array}{l}-0.053[-0.116-0.017] \\
p=0.359(n=9)\end{array}$ & $p=0.010$ \\
\hline & Beta [12-30 Hz] & $\begin{array}{l}-0.221[-0.288--0.150] \\
p=0.004(n=10)\end{array}$ & $\begin{array}{l}-0.069[-0.112-0.001] \\
p=0.055(n=9)\end{array}$ & $\mathrm{p}=0.017$ \\
\hline & Gamma [30-100 Hz] & $\begin{array}{l}-0.072[-0.119--0.013] \\
p=0.027(n=10)\end{array}$ & $\begin{array}{l}0.003[-0.043-0.033] \\
p=1(n=9)\end{array}$ & $\mathrm{p}=0.065$ \\
\hline
\end{tabular}

S7 Table (related to Fig 5E). MI of power after C21 injection.

\begin{tabular}{|c|c|c|c|c|}
\hline & & $\begin{array}{l}\text { Baseline } \\
\text { (median, interquartile } \\
\text { range, in \%) }\end{array}$ & $\begin{array}{l}\text { Compound } 21 \\
\text { (median, interquartile range, in } \\
\%)\end{array}$ & $\begin{array}{l}\mathbf{p} \\
\text { (Wilcoxon } \\
\text { signed-rank } \\
\text { test) }\end{array}$ \\
\hline \multirow[t]{2}{*}{ OB } & $\mathrm{Cre}^{+}(\mathrm{n}=17)$ & $26.131[20.490-32.841]$ & $19.083[2.638-24.127]$ & $p=0.031$ \\
\hline & Cre $^{-}(n=17)$ & $32.310[20.032-37.206]$ & $31.103[22.888-51.465]$ & $p=0.435$ \\
\hline \multirow[t]{2}{*}{ LEC } & $\mathrm{Cre}^{+}(\mathrm{n}=13)$ & $48.216[35.999-53.788]$ & 14.889 [6.576 - 28.939] & $p=0.0005$ \\
\hline & Cre $^{-}(n=11)$ & 48.294 [29.988 - 59.585] & $41.896[23.372-52.576]$ & $p=0.147$ \\
\hline \multirow[t]{2}{*}{ HP } & $\mathrm{Cre}^{+}(\mathrm{n}=10)$ & $40.596[36.197-53.448]$ & $18.349[9.143-33.924]$ & $p=0.002$ \\
\hline & Cre- $^{-}(n=9)$ & $53.911[44.874-64.950]$ & $43.233[30.020-60.254]$ & $\mathrm{p}=0.164$ \\
\hline
\end{tabular}

S8 Table (related to Fig 5F and S3B Fig). Time in discontinuous events. 


\begin{tabular}{lllll} 
& & $\begin{array}{l}\text { Cre }^{+} \\
\text {(median, interquartile } \\
\text { range) } \\
\text { nunits=415 from 15 mice }\end{array}$ & $\begin{array}{l}\text { Cre } \\
\text { (median, interquartile } \\
\text { range) } \\
\text { nunits=361 from 16 mice }\end{array}$ & $\begin{array}{l}\mathrm{p} \\
\text { (linear mixed- } \\
\text { effect model) }\end{array}$ \\
\hline OB & $0.230[0.096-0.367]$ & $0.036[-0.074-0.160]$ & $\mathrm{p}=0.351$ \\
\cline { 2 - 5 } & RR [2-4 Hz] & $-0.025[-0.189-0.056]$ & $-0.014[-0.098-0.093]$ & $\mathrm{p}=0.017$ \\
\hline & Theta [4-12 Hz] & $-0.184[-0.368--0.075]$ & $-0.041[-0.121-0.009]$ & $\mathrm{p}=0.0002$ \\
\hline Beta [12-30 Hz] & $-0.103[-0.195-0.016]$ & $-0.020[-0.071-0.044]$ & $\mathrm{p}=0.005$
\end{tabular}

S9 Table (related to S4A Fig). STP for OB after C21 injection.

\begin{tabular}{|c|c|c|c|c|}
\hline & & $\begin{array}{l}\mathrm{Cre}^{+} \\
\text {(median, interquartile } \\
\text { range, Wilcoxon signed- } \\
\text { rank test) }\end{array}$ & $\begin{array}{l}\text { Cre- } \\
\text { (median, interquartile } \\
\text { range, Wilcoxon signed- } \\
\text { rank test) }\end{array}$ & $\begin{array}{l}\mathbf{p} \\
\text { (Wilcoxon } \\
\text { rank-sum } \\
\text { test) }\end{array}$ \\
\hline \multirow[t]{4}{*}{ OB-LEC } & RR [2-4 Hz] & $\begin{array}{l}-0.052[-0.208-0.150] \\
p=1(n=13)\end{array}$ & $\begin{array}{l}-0.103[-0.368-0.212] \\
p=1(n=11)\end{array}$ & $p=0.908$ \\
\hline & Theta [4-12 Hz] & $\begin{array}{l}-0.278[-0.476-0.213] \\
p=1(n=13)\end{array}$ & $\begin{array}{l}-0.074[-0.218-0.083] \\
p=0.549(n=11)\end{array}$ & $\mathrm{p}=0.862$ \\
\hline & Beta [12-30 Hz] & $\begin{array}{l}-0.239[-0.346--0.051] \\
p=0.022(n=13)\end{array}$ & $\begin{array}{l}0.005[-0.119-0.226] \\
p=0.549(n=11)\end{array}$ & $p=0.013$ \\
\hline & Gamma [30-100 Hz] & $\begin{array}{l}-0.021[-0.109-0.151] \\
p=0.581(n=13)\end{array}$ & $\begin{array}{l}-0.021[-0.124-0.330] \\
p=1(n=11)\end{array}$ & $p=0.524$ \\
\hline \multirow[t]{4}{*}{ LEC-HP } & RR [2-4 Hz] & $\begin{array}{l}-0.072[-0.268-0.528] \\
p=0.727(n=8)\end{array}$ & $\begin{array}{l}0.024[-0.089-0.251] \\
p=1(n=7)\end{array}$ & $p=0.867$ \\
\hline & Theta [4-12 Hz] & $\begin{array}{l}-0.082[-0.436-0.092] \\
p=0.727(n=8)\end{array}$ & $\begin{array}{l}-0.176[-0.304--0.056] \\
0.125(n=7)\end{array}$ & $\mathrm{p}=0.955$ \\
\hline & Beta [12-30 Hz] & $\begin{array}{l}-0.095[-0.214--0.026] \\
p=0.070(n=8)\end{array}$ & $\begin{array}{l}-0.147[-0.211--0.024] \\
p=0.125(n=7)\end{array}$ & $p=0.779$ \\
\hline & Gamma [30-100 Hz] & $\begin{array}{l}-0.079[-0.183-0.133] \\
p=0.727(n=8)\end{array}$ & $\begin{array}{l}-0.022[-0.053-0.090] \\
p=1 \quad(n=7)\end{array}$ & $p=0.536$ \\
\hline \multirow[t]{4}{*}{ OB-HP } & RR [2-4 Hz] & $\begin{array}{l}0.311[0.214-0.501] \\
p=0.109(n=10)\end{array}$ & $\begin{array}{l}0.169[-0.303-0.212] \\
p=1(n=9)\end{array}$ & $p=0.182$ \\
\hline & Theta [4-12 Hz] & $\begin{array}{l}0.045[-0.174-0.341] \\
p=0.754(n=10)\end{array}$ & $\begin{array}{l}-0.047[-0.204--0.006] \\
p=0.180(n=9)\end{array}$ & $p=0.356$ \\
\hline & Beta [12-30 Hz] & $\begin{array}{l}-0.318[-0.397--0.124] \\
p=0.021(n=10)\end{array}$ & $\begin{array}{l}-0.178[-0.306--0.058] \\
p=0.039(n=9)\end{array}$ & $p=0.278$ \\
\hline & Gamma [30-100 Hz] & $\begin{array}{l}0.036[-0.067-0.161] \\
p=1(n=10)\end{array}$ & $\begin{array}{l}-0.074[-0.096-0.060] \\
p=0.508(n=9)\end{array}$ & $p=0.095$ \\
\hline
\end{tabular}

S10 Table (related to Fig 6B). MI coherence for cre $^{+}$and cre- mice. 


\begin{tabular}{lllll} 
& & $\begin{array}{l}\text { Area 1 } \rightarrow \text { Area 2 } \\
\text { (median, interquartile } \\
\text { range })\end{array}$ & $\begin{array}{l}\text { Area 2 } \rightarrow \text { Area 1 } \\
\text { (median, interquartile } \\
\text { range })\end{array}$ & $\begin{array}{l}\mathrm{p} \\
\text { (Wilcoxon } \\
\text { signed-rank test })\end{array}$ \\
\hline $\begin{array}{l}\text { Area1: } \\
\text { OB }\end{array}$ & Baseline Cre+ $(\mathrm{n}=13)$ & $2.164[1.769-2.777]$ & $0.366[0.249-0.433]$ & $\mathrm{p}=0.0002$ \\
\cline { 2 - 5 } $\begin{array}{l}\text { Area2: } \\
\text { LEC }\end{array}$ & C21 Cre+ $(\mathrm{n}=13)$ & $1.211[0.871-1.666]$ & $0.600[0.378-0.855]$ & $\mathrm{p}=0.080$ \\
\cline { 2 - 5 } & Baseline Cre- $(\mathrm{n}=11)$ & $1.616[0.738-2.474]$ & $0.494[0.345-1.228]$ & $\mathrm{p}=0.102$ \\
\cline { 2 - 5 } & C21 Cre- $(\mathrm{n}=11)$ & $1.628[1.062-2.802]$ & $0.487[0.271-0.673]$ & $\mathrm{p}=0.042$ \\
\hline $\begin{array}{l}\text { Area1: } \\
\text { LEC }\end{array}$ & Baseline Cre+ $(\mathrm{n}=8)$ & $0.705[0.531-0.986]$ & $1.064[0.709-1.507]$ & $\mathrm{p}=0.195$ \\
\cline { 2 - 5 } $\begin{array}{l}\text { Area2: } \\
\text { HP }\end{array}$ & C21 Cre+ $(\mathrm{n}=8)$ & $0.683[0.534-0.896]$ & $1.202[0.806-1.271]$ & $\mathrm{p}=0.383$ \\
\cline { 2 - 5 } & Baseline Cre- $(\mathrm{n}=7)$ & $0.607[0.597-0.773]$ & $0.928[0.563-1.171]$ & $\mathrm{p}=0.469$ \\
\cline { 2 - 5 } & C21 Cre- $(\mathrm{n}=7)$ & $0.634[0.436-0.841]$ & $1.072[0.510-1.528]$ & $\mathrm{p}=0.469$ \\
\hline $\begin{array}{l}\text { Area1: } \\
\text { OB }\end{array}$ & Baseline Cre+ $(\mathrm{n}=10)$ & $1.422[1.257-2.076]$ & $0.492[0.367-0.618]$ & $\mathrm{p}=0.002$ \\
\cline { 2 - 5 } $\begin{array}{l}\text { Area2: } \\
\text { HP }\end{array}$ & C21 Cre+ $(\mathrm{n}=10)$ & $1.107[0.709-1.605]$ & $0.531[0.430-1.012]$ & $\mathrm{p}=0.625$ \\
\cline { 2 - 5 } & Baseline Cre- $(\mathrm{n}=9)$ & $1.703[1.181-1.752]$ & $0.349[0.322-0.623]$ & $\mathrm{p}=0.012$ \\
\cline { 2 - 5 } & C21 Cre- $(\mathrm{n}=9)$ & $1.469[1.145-2.010]$ & $0.304[0.292-0.624]$ & $\mathrm{p}=0.012$
\end{tabular}

S11 Table (related to Fig 6D and S5B Fig). SDR before and after C21 injection.

Baseline (median, interquartile range)

\section{Compound 21}

(median, interquartile range)
(Wilcoxon signed-rank test)

\begin{tabular}{lllll}
\hline Area1: OB & & & & test \\
Area2: LEC & $\begin{array}{l}\text { Difference } \\
\text { Cre+ }(n=13)\end{array}$ & $1.792[1.300-2.512]$ & $0.619[0.087-1.160]$ & $\mathrm{p}=0.001$ \\
\cline { 2 - 5 } & $\begin{array}{l}\text { Difference } \\
\text { Cre- }(\mathrm{n}=11)\end{array}$ & $1.192[-0.490-2.130]$ & $1.045[0.479-2.531]$ & $\mathrm{p}=0.175$ \\
\hline Area1: LEC & $\begin{array}{l}\text { Difference } \\
\text { Crea }(\mathrm{n}=8)\end{array}$ & $-0.357[-0.929-0.254]$ & $-0.471[-0.746-0.041]$ & $\mathrm{p}=0.641$ \\
\cline { 2 - 5 } & $\begin{array}{l}\text { Difference } \\
\text { Cre- }(\mathrm{n}=7)\end{array}$ & $-0.326[-0.582-0.210]$ & $-0.438[-1.092-0.331]$ & $\mathrm{p}=0.813$ \\
\hline Area1: OB & $\begin{array}{l}\text { Difference } \\
\text { Area2: HP }\end{array}$ & $0.891[0.601-1.709]$ & $0.627[-0.303-1.175]$ & $\mathrm{p}=0.004$ \\
\cline { 2 - 5 } & $\begin{array}{l}\text { Cifference } \\
\text { Cre- }(\mathrm{n}=9)\end{array}$ & $1.347[0.557-1.413]$ & $1.167[0.553-1.708]$ & $\mathrm{p}=0.496$
\end{tabular}

S12 Table (related to Fig 6D). SDR difference for before and after C21 injection. 